\title{
Functional and Biomechanical Effects of the Edge-to-Edge Repair in the Setting of Mitral Regurgitation: Consolidated Knowledge and Novel Tools to Gain Insight into Its Percutaneous Implementation
}

Francesco Sturla 1,2

Alberto Redaelli 1

Giovanni Puppini 2

Francesco Onorati 2

Giuseppe Faggian 2

Emiliano Votta $1{ }^{*}$

Phone +39-02-2399-3377

Emailemiliano.votta@polimi.it

1 Department of Electronics, Information and Bioengineering (DEIB), Politecnico di Milano, Via Golgi 39, 20133 Milan, Italy

2 Division of Cardiovascular Surgery, Università degli Studi di Verona, Verona, Italy

\section{Abstract}

Mitral regurgitation is the most prevalent heart valve disease in the western population. When severe, it requires surgical treatment, repair being the preferred option. The edge-to-edge repair technique treats mitral regurgitation by suturing the leaflets together and creating a double-orifice valve. Due to its relative simplicity and versatility, it has become progressively more widespread. Recently, its percutaneous version has become feasible, and has raised interest thanks to the 
positive results of the Mitraclip ${ }^{\circledR}$ device. Edge-to-edge features and evolution have stimulated debate and multidisciplinary research by both clinicians and engineers. After providing an overview of representative studies in the field, here we propose a novel computational approach to the most recent percutaneous evolution of the edge-to-edge technique. Image-based structural finite element models of three mitral valves affected by posterior prolapse were derived from cine-cardiac magnetic resonance imaging. The models accounted for the patient-specific 3D geometry of the valve, including leaflet compound curvature pattern, patient-specific motion of annulus and papillary muscles, and hyperelastic and anisotropic mechanical properties of tissues. The biomechanics of the three valves throughout the entire cardiac cycle was simulated before and after Mitraclip ${ }^{\circledR}$ implantation, assessing the biomechanical impact of the procedure. For all three simulated MVs, Mitraclip ${ }^{\circledR}$ implantation significantly improved systolic leaflets coaptation, without inducing major alterations in systolic peak stresses. Diastolic orifice area was decreased, by up to $58.9 \%$, and leaflets diastolic stresses became comparable, although lower, to systolic ones. Despite established knowledge on the edge-to-edge surgical repair, latest technological advances make its percutanoues implementation a challenging field of research. The modeling approach herein proposed may be expanded to analyze clinical scenarios that are currently critical for Mitraclip ${ }^{\circledR}$ implantation, helping the search for possible solutions.

\section{Keywords}

Mitral valve

Mitral regurgitation

Heart valve biomechanics

Edge-to-edge

Mitraclip

Finite element model

Associate Editor Karyn Kunzelman oversaw the review of this article.

\section{Introduction}

\section{The Mitral Valve}


The mitral valve (MV) separates the left atrium (LA) from the left ventricle (LV). It consists of four substructures: mitral annulus (MA), anterior and posterior leaflets, inserted onto the MA, two papillary muscles (PMs) inserted in the LV myocardium, and chordae tendineae connecting the leaflets to the PMs. In healthy conditions, the MV guarantees the unidirectional blood flow from the LA to the LV during diastolic ventricular filling, and prevents from backflows into the LA during systolic ventricular ejection. During diastole, the MA area widens, PMs are relaxed and the MV opens under the action of minimal trans-mitral pressure. In systole, the sphincteric action of the surrounding myocardium shrinks and bends the MA, reducing its perimeter and area, while PMs contract and tighten the chordae tendineae. This mechanism allows for the correct coaptation of MV leaflets and for competence of the valve.

\section{Mitral Regurgitation}

As for any heart valve, the MV can become stenotic or regurgitant in pathologic conditions. Mitral regurgitation (MR) in particular is the most prevalent heart valve disease in the western population. 58 Two main types of MR can be identified: the less-frequently observed type at surgery is functional MR, which is secondary to LV geometrical distortions that prevent from MV continence despite the integrity of valvular substructures. However, given the epidemic nature of heart failure in the last decades - which is estimated to affect up to date 6 million patients in US only-it is possible that functional MR is currently underestimated in clinical practice, and that it will represent the most commonly observed type of MR of the next decades. 3 Typically, functional MR is characterized by dislocation of one or both PMs away from the valvular orifice, with associated tethering of chordae tendineae and leaflets insufficient mobility, function even if the latter is structurally physiological. 18 The most common one is degenerative MR, which is associated to structural alterations MV substructures, usually leaflets and/or chordae tendineae, possibly followed by annular dilation. 2 In particular, the most common mitral disease in western Countries is degenerative mitral valve (DMV) prolapse, whose most frequent distinctive pathologic feature is posterior leaflet prolapse.

\section{Surgical Setting of Mitral regurgitation}

When severe, mitral regurgitation heavily reduces life expectancy and 
quality of life, and, according to current guidelines, it should be surgically treated when symptoms are evident 78 or when progressive LV dilation/dysfunction and/or pulmonary hypertension develop, regardless of symptoms. 9

Surgical repair and replacement are the available options; the former is currently considered superior to the latter, in particular in the treatment of degenerative MR, 15 and is strongly recommended over replacement in both European and US current Guidelines. 9,78

Different paradigms of surgical repair approach exist. The Carpentier's techniques, which have been considered the standard of care for decades until the advent of ePTFE sutures and chordal repair techniques, aim at reconstructing the diseased MV towards a physiologic-like morphology through the combination of annuloplasty, i.e., the reshaping of the native MA by implanting a prosthetic ring, resection of abnormal or pathologic leaflet tissue, and chordal transfer or replacement. These techniques are well established and associated with excellent long-term outcomes, 11,23 however their implementation can be complex and operator-dependent in the setting of the most complex pathological scenarios. More recently, the "respect rather than resect" paradigm has emerged, and is based on the use of polytetrafluoroethylene (PTFE) neochordae to reconstruct support of the free edge of prolapsing segments avoiding the resection of leaflet tissue. 19

\section{The Edge-to-Edge Technique}

\section{The Standard Surgical Approach}

A third and completely different concept characterizes the Alfieri-stitch technique, or edge-to-edge technique, initially developed by Alfieri in the early 1990s 24,47 : while the previously mentioned approaches aim at restoring competence through the reconstruction of a "morphologically normal" $\mathrm{MV}$, this technique aims at restoring competence through a "functional" rather than anatomical repair. The edge-to-edge technique mainly consists in accurately identifying the location of the regurgitant jet, and in suturing the free edge of the prolapsing sector of the diseased leaflet to the corresponding edge of the complementary leaflet exactly where the jet was observed. As a result, when the sutured sector is in the central part of the MV (i.e., A2 and P2 scallops), a double orifice is obtained; the two orifices can have similar or significantly different extent, depending on the 
position and extent of the suture. When the sutured sector is next to a commissure (i.e., A1-P1 and A3-P3 scallops), a single orifice is maintained. 5 However, according to the prevalent incidence of A2 and/or P2 prolapse in human pathology, the "double-orifice" configuration prevail in current surgical practice. In any case, the total area available for diastolic trans-mitral flow is reduced as compared to pre-operative conditions, with reductions up to $50 \%$ of the initial area. Hence, the success of the procedure in degenerative MR depends on two key aspects: the correct identification of the regurgitant jet, and the achievement of a suitable trade-off between the need for restraining the originally prolapsing leaflet and the need for avoiding excessive reduction in MV orifice area and associated risks of stenosis.

If these two criteria are fulfilled, the edge-to-edge allows for success through a technically simple, reproducible and versatile surgical maneuver. However, the edge-to-edge technique is currently suggested also in patients with functional MR, though the key-to-success tips are the correct identification of the regurgitant jet at preoperative echocardiography (in order to guide the intraoperative stitching of the two mitral leaflets) and the constant association of a two-size undersizing annuloplasty with a complete rigid (or at least semirigid) prosthetic ring. 4

After being long debated within the scientific community, thanks to the abovementioned features this technique has become increasingly adopted in a wide spectrum of pathological scenarios, although when annular dilation (e.g., in case of functional MR and in case of Barlow disease) or multiple jets are present the edge-to-edge technique alone is not sufficient and needs a complementary mitral annuloplasty, in order to improve longterm durability of the repair. $8,49,50$

\section{From the Standard Surgical Approach to Percutaneous Procedures}

Thanks to its simplicity, and to the possibility to be adopted as standalone approach in some clinical scenarios, in the last decade the edge-to-edge technique has become a target for the recent transition from classical openchest surgery towards minimally invasive surgery and, lastly, percutaneous interventions. 51

Initial attempts to reproduce the edge-to-edge technique via percutaneous 
approach were made with different technological solutions: since the encouraging in vitro results obtained with a grasper (to coapt leaflets) and a spiral screw (to fasten them), 55 Alfieri and colleagues successfully performed the edge-to-edge technique by the aid of a suctioning and stitching device, on an "off-pump" beating heart ovine model. 6 A similar solution (Mobius, Edwards Lifesciences LLC, Irvine, CA, USA) was successfully tested in an acute animal study, 56 but subsequent clinical trials proved it insufficiently effective and led to its rejection. 82

A different solution was implemented into Mitraclip ${ }^{\circledR}$ system (Abbot Vascular, Inc., Menlo Park, California, US), which is the only percutaneous device for edge-to-edge currently approved for clinical use in humans, and has become the one most widely adopted transcatether device, with more than 16,000 patients treated worldwide since its first implantation in 2003.20 This device comprises a mechanical clip, approximately $4 \mathrm{~mm}$ wide and $10 \mathrm{~mm}$ long, mounted on the distal end of a clip delivery system, and a steerable guide catheter (Fig. 1). Under general anesthesia and with fluoroscopic and transesophageal echocardiographic guidance, the system gains access to the LA through standard trans-septal puncture. The clip is subsequently guided into the LV across the mitral orifice with its arms in open position, and is hence pulled back and positioned to capture the MV leaflets in the region where the regurgitant jet was observed. The clip arms are then closed, and MV leaflet edges are grasped by means of ad hoc barbs. Prior to its final release, the clip can be repositioned and readjusted, if needed (Fig. 1). Different clinical trials have evaluated this device. $10,21,22,61,73,83$ In particular, the EVEREST I, EVEREST II, and EVEREST High Risk Registry multicenter studies compared the outcomes of the Mitraclip ${ }^{\circledR}$ procedure vs. standard surgical mitral repair in hundreds of patients. $21,22,83$ EVEREST I and EVEREST II involved patients with moderate-severe or severe MR, mainly with degenerative aetiology, who respected the following inclusion criteria at echocardiographic examination: in case of leaflet prolapse, residual coaptation length $\geq 2 \mathrm{~mm}$ and coaptation depth $<11 \mathrm{~mm}$; in case of leaflet flail, i.e., complete eversion of the leaflet into the LA, flail gap $<10 \mathrm{~mm}$ and flail width $<15 \mathrm{~mm}$. These two studies established that (i) the device and the procedure are feasible, (ii) the device is effective in reducing MR (post-operative grade $\leq 2$ ) in the majority of cases as long as MV anatomy is carefully evaluated prior to implantation, (iii) it is associated with lower periprocedural complications, but also to 
inferior efficacy, as compared to standard surgery. 22 Patients enrolled in the EVEREST High Risk Registry were at high surgical risk due to age and comorbidities, and were almost evenly distributed between the two types of MR. In this specific subgroup, mortality associated to Mitraclip ${ }^{\circledR}$ implantation was lower as compared to standard surgery, 1-year follow-up survival was higher and rate of rehospitalization was lower. Moreover, the device proved able to improve patients' functional class and quality of life independently of MR aetiology.

\section{Figure 1}

Mitraclip ${ }^{\circledR}$ device and procedure. (a) Section of the human heart, showing the access of the Mitraclip ${ }^{\circledR}$ device into the left atrium through transseptal puncture; RA, right atrium; RV, right ventricle; LA, left atrium; LV, left ventricle; AV, aortic valve; TV, tricuspid valve; MV, mitral valve; MC, Mitraclip ${ }^{\circledR}$ device. (b) The device is guided into the LV and positioned below the regurgitant region of the MV, with its arms open. (c) The device is pulled up, the leaflets are grasped and the arms are closed. (d) The clip is released and the delivery system is retrieved 


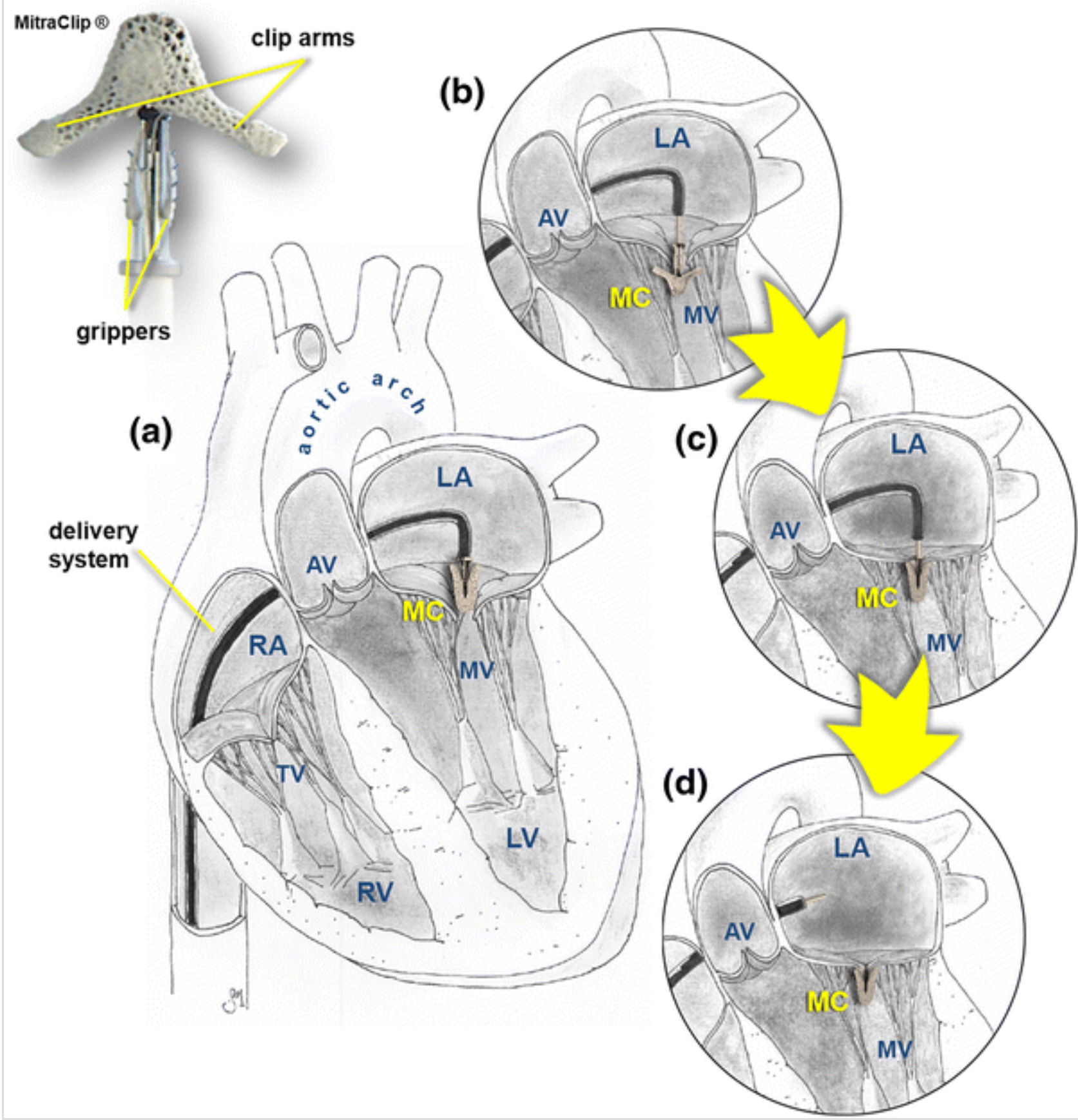

However, despite its conceptual ease, mitraclip implantation still represents a complex procedure, requiring skilled operators, experienced echocardiographists and a high-level overall clinical staff and environment (i.e., the procedure can't be routinely managed in all hospitals). In addition, such as other percutaneous techniques (e.g., transcatheter aortic valve implantation), a demanding learning curve is necessary in order to efficiently perform the procedure and acquire a high-quality level of implantation through a long-lasting experience.

\section{Acquired Knowledge on the Mechanisms Underlying MV Function Following Edge-to- Edge Technique}


The debate characterizing the initial diffusion of the edge-to-edge technique, the analysis of its potential drawbacks, and the path towards its implementation via percutaneous procedures has raised the increasing attention of the medical and engineering communities, as testified by the amount of scientific publications on the topic. A simple search with the keywords "mitral edge-to-edge" and "mitraclip" in the Pubmed database (http://www.ncbi.nlm.nih.gov/pubmed/) allowed to retrieve about 300 publications on this specific topic since 1998-2013, with a rather impressive increasing trend in most recent years, associated to studies focused on the percutaneous approach (Fig. 2). Along with manuscripts reporting clinical outcomes, these studies include work aim at quantifying the biomechanical effects of the edge-to-edge technique through animal models, in vitro analyses, and computational models based on numerical techniques. The heterogeneity of these approaches reflects the need for a multidisciplinary vision to understand complex issues such as the effects of MV repair techniques. In the following, an overview of relevant and representative studies is provided; these are grouped based on the specific aspect they try to elucidate regarding the impact of the edge-to-edge technique: (i) MV dynamic geometry and hemodynamics, (ii) LV fluiddynamics, and (iii) structural effects.

\section{Figure 2}

Number of publications since 1998-2013 retrieved from the Pubmed database by searching for the keywords "mitral edge-to-edge" (green) and "mitraclip" (red) 


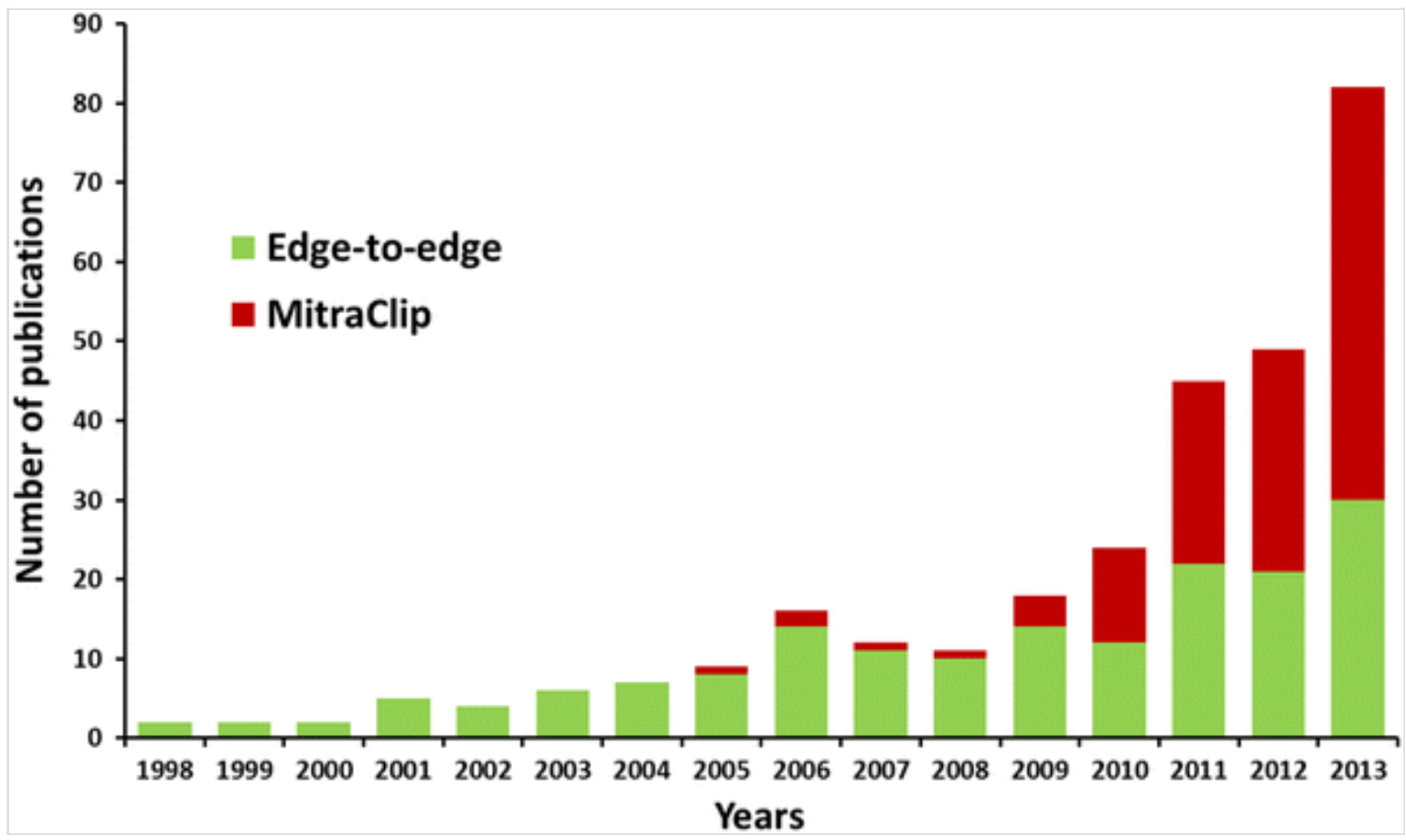

\section{Effects on MV Geometry and Hemodynamics}

As previously mentioned, the edge-to-edge technique restores the function of the MV, but imposes a non-physiologic configuration of the MV leaflets. Hence, negative effects on MV geometry and hemodynamics have been hypothesized.

As regards geometry, based on the tight inter-relation between MV substructures, possible indirect effects on the annulus and on the subvalvular apparatus, i.e., chordae tendineae and PMs, have been speculated. Timek and colleagues quantified these possible effects in ovine models. 75,76 After surgical implantations of radiopaque micromarkers on LV endocardium, MA, and leaflets free edge, animal were kept intubated, anesthetized, and with open chest. The authors acquired markers 3D position via biplane videofluoroscopy, hemodynamic data, and epicardial color Doppler echocardiography in different conditions. When the edge-toedge repair was performed, the A2 and P2 cusps were stitched together, and the stitch was instrumented with a load transducer. In, 75 they compared physiologic-like conditions vs. post edge-to-edge repair with and without inotropic stimulation, and observed that in no case the repair procedure significantly affected annular dynamics. These results showed that the tension of the stitch does not indirectly impair annular dynamics, and alleviated the concern regarding the risk for functional mitral stenosis during exercise. Similar conclusions were drawn by Hasegawa and co- 
workers based on an in vitro study performed on isolated and pulsatile swine hearts, 27 which showed no statistically significant difference between MA area before and after edge-to-edge repair, as measured on a 2D projection from high-speed digital video camera acquisitions performed through endoscopic apical access to the LV.

On the other hand in Ref. 76, Timek and co-workers analyzed the effects of the edge-to-edge technique in physiologic-like conditions and after inducing acute ischemic mitral regurgitation (IMR) through proximal left circumflex occlusion. They showed that (i) in absence of IMR the repair slightly limited annular dynamics, (ii) during IMR annular diameters and area increased and lost their baseline dynamic variations, while the distance between PMs and MA increased, (iii) the geometrical distortions induced by IMR were not affected by the edge-to-edge repair. These results suggested that the stitch should not indirectly alter the geometry of the sub-valvular apparatus, but at the same time could not counteract the geometrical effects of LV ischemia.

As regards hemodynamics, the reduction of the area available for diastolic trans-mitral flow has raised questions regarding the risk of stenosis. However, this concern was mitigated by different studies. In one of the previously mentioned animal studies, 75 mean diastolic trans-mitral pressure drop was $0.7 \pm 0.4 \mathrm{mmHg}$ in physiologic-like conditions; after edge-to-edge repair, it increased to $1.4 \pm 0.4$ and $2.4 \pm 1.0 \mathrm{mmHg}$ prior to and after dobutamine infusion, which induced a $89 \%$ increase in cardiac output. Hence, the mean diastolic pressure drop remained lower than $3 \mathrm{mmHg}$ in every condition, despite the over $200 \%$ increase in stress conditions. Similar trends were observed for the peak diastolic trans-mitral pressure drop, which remained below $5 \mathrm{mmHg}$ even in stress conditions after edge-to-edge repair.

In a recent clinical study, the records and intraoperative transesophageal echocardiographic data of 552 consecutive patients affected by MR, of whom 84 being treated though edge-to-edge repair, were analyzed.29 Peak and mean trans-mitral pressure drop following edge-to-edge repair were significantly higher as compared to standard repair techniques (peak values: $10.7 \pm 0.5$ vs. $7.1 \pm 0.2 \mathrm{mmHg}$, mean values: $4.3 \pm 0.2$ vs. $2.8 \pm 0.1 \mathrm{mmHg}$ ), although within the threshold values that are usually tolerated by patients. Also, trans-mitral pressure drop data obtained in 
edge-to-edge patients with and without annuloplasty did not vary significantly. This experimental evidence is also confirmed by in vitro 27 and computational fluid-dynamics (CFD) analyses. 48 In Ref. 27, Hasegawa and co-workers also acquired volumetric data, and showed that the edge-to-edge suture did not induce mitral stenosis despite a significant (30\%) reduction in effective orifice area. In Ref. 48, Maisano and colleagues reported that for an effective orifice area of $3 \mathrm{~cm}^{2}$ and with a flow rate of $11 \mathrm{~L} / \mathrm{min}$ the peak diastolic trans-mitral pressure drop does not exceed $5 \mathrm{mmHg}$, and only when the effective orifice area falls below $2.5 \mathrm{~cm}^{2}$ a steep increase in trans-mitral pressure drop is computed.

As for the analyses focused on the geometrical effects of the edge-to-edge repair, those focused on the hemodynamic effects highlighted some limitations of the repair technique in the the setting of functional MR as compared to degenerative MR, and the need for concomitant annuloplasty in the former case. Sheep models showed no significant difference in terms of degree of regurgitation between repaired and non-repaired valves during acute IMR.76 In an elegant in vitro study, Croft and colleagues simulated IMR with and without annular dilation, before and after edge-to-edge repair, on porcine isolated valves mounted in a pulsatile mock loop comprising a left-heart simulator (GeorgiaTech Left Heart Simulator) and instrumented with pressure and flow transducers. 13 The experimental setup allowed for tuning PMs position in the 3D space by means of ad hoc actuators and annular dimensions by means of a draw string mechanism. Experiments were run with three annular configurations (baseline, and 56 and $120 \%$ annular area increase), and for each annular configuration symmetrical and non-symmetrical PMs dislocation was imposed by displacing the target PM by $10 \mathrm{~mm}$ in the apical, lateral and posterior direction. Comparison of regurgitant volumes pre- and post-edge-to-edge repair was carried out for each combination of annulus and PMs configurations: statistically significant differences in presence of PMs dislocation were observed only if annular dilation was also simulated; reductions in regurgitant volume associated to edge-to-edge repair were always below $11 \%$, and could not eliminate MR.

\section{Effects on LV Fluid-Dynamics}

MV and LV function are strictly related. On the one hand, due to their anatomical continuity, LV geometrical distortions, as the ones 
characterizing functional MR, can impair MV function, while alterations of the MV, such as the cutting of chordae tendineae in the replacement of the native valve with a prosthesis, can lead to LV dilation. 66 On the other hand, MV dynamics and intracavitary LV fluid-dynamics influence each other: for instance, the geometry of MV leaflets influences the fluiddynamics in the LV outflow tract during systolic ejection, 16 as well as the physiological formation of vortex structures in the LV during diastolic filling, 33 which is related to energy recovery in the LV from diastole to systole, and hence to the efficiency of LV pumping function. Since the edge-to-edge repair creates a double-orifice MV, different research groups have quantified its impact on diastolic LV filling, usually by means of CFD or fluid-structure interaction (FSI) models.

The aforementioned CFD study by Maisano and colleagues, 48 described the LA as a cylinder, the LV as a cone frustum, and the surface of MV leaflets as concave towards the LA. Under these assumptions, it investigated LV fluid-dynamics in three leaflet configurations: (i) a Dshaped orifice, resembling the result of simple annuloplasty with a complete rigid ring, (ii) two equal and symmetrical orifices, resembling the outcome of central edge-to-edge repair, and (iii) two orifices with different extent (2:1 area ratio), simulating the result of edge-to-edge repair with asymmetrical suture. In all of the three configurations, the total orifice area was the same, and its value was set to $1.5,2.25$ and $3 \mathrm{~cm}^{2}$. Transient simulations with a maximum flow rate of $11 \mathrm{~L} / \mathrm{min}$ were run for each geometry, and the influence of flow rate $(7,11,15 \mathrm{~L} / \mathrm{min})$ was assessed only with the symmetrical double orifice and a total area of $2.25 \mathrm{~cm}^{2}$. The study reported that for any given total orifice area, the maximum blood velocity and the trans-mitral pressure drop did not depend on the specific configuration of the orifice. Also, even if non-symmetrical, the two orifices created by the edge-to-edge repair are characterized by the same local maximum velocity. Finally, for every edge-to-edge configuration, the fluid-dynamics within the ventricle was characterized by the presence of jets originating at the orifices, directed apically and with increasing extent when smaller orifice area were considered, as well as by equivalent pressure fields. These were characterized by a pressure drop in the upper half of the LV associated to flow vortexes, whose entity was similar as compared to the one computed with a single MV orifice, and by a pressure recovery towards the apical region, which was about $20 \%$ of the maximum trans-mitral pressure drop, and indicated that not all of the kinetic energy 
associated to the jets is dissipated.

Through a geometrically more complex model, which included an almost spherical LV, a reasonably realistic mutual positioning of MV and aortic valve, as well as PMs, $\mathrm{Hu}$ and colleagues analyzed the same issue in presence of central and lateral edge-to-edge suture. 17,30 With respect to, 48 in $\mathrm{Hu}$ et al. 30 they provided extra insight by analyzing the 3D vortex structures in the LV associated to the double-orifice MV. In physiological conditions, LV filling is characterized by the formation of a distinct vortex ring surrounding the incoming blood jet, beneath the MV. This vortex then develops from the MV anterior leaflet and moves down towards the LV apex, while the blood jet is redirected towards the LV outflow tract. In presence of central edge-to-edge repair, the large vortex structure by the MV anterior leaflet was lost; instead, two vortex rings surrounded the two jets, which originated from the double orifice and were directed towards the PMs. The impingement of the two jets against the LV wall was the main cause of energy dissipation, while the vortex structure, although more chaotic as compared to physiological conditions, contributed to dissipation to a minor extent. Overall, the energy dissipation associated to edge-to-edge correction depended on the double-orifice total area, and was 2.5 times greater than the physiological one when the greatest double-orifice total area was considered (i.e., $87 \%$ of the physiological, single orifice area). These results were confirmed by a more complex FSI model of diastolic MV function in presence of central edgeto-edge repair. 44

\section{Structural Effects on the MV}

One of the potential drawbacks of the edge-to-edge technique consists in the extra tensions induced by the stitch on MV leaflets. Hence, several studies have been carried out to quantify such tensions, and to assess its dependency on pre-operative MV geometry and on hemodynamics parameters.

In general, the direct measurement of leaflets stresses in complex structure such as the MV is practically impossible: at best, these can be indirectly estimated from measured strains through the use of constitutive models and numerical techniques. 45 For this reason, experimental studies on this specific aspect were focused on the measurement of the stitch tension, which, due to equilibrium conditions, is transmitted to the leaflets 
generating stresses.

Nielsen and colleagues 57 analyzed the relationship between stitch tension and MV geometry in an ovine model, following the same approach adopted in Timek et al. 76; after acquiring hemodynamic data in physiologic conditions and during circumflex artery occlusion, they implanted tantalum markers on MA, leaflets free edge, PMs and LV endocardium, and performed a central edge-to-edge repair instrumenting the suture with a miniature force transducer. Hemodynamic measurements were then repeated, stitch tension was recorded, and markers' coordinates were acquired through biplane fluoroscopy with and without circumflex artery occlusion. The analysis of the time-dependent measured variables showed that the edge-to-edge repair had a small effect on the acute ischemic MR associated to circumflex artery occlusion, and that stitch tension had no relation to the degree of generated MR. Peak stitch tension was detected in diastole, when the septo-lateral dimension of the MA was maximal; the time-course of stitch tension appeared not synchronized with the timecourse of the distance between leaflets free edge. Acute ischemic MR induced a statistically relevant increase in stitch tension (from $0.26 \pm 0.05$ to $0.46 \pm 0.08 \mathrm{~N}$ ) and annulus septo-lateral dimension (from $29.8 \pm 1.4$ to $31.2 \pm 1.3$ ), but not in leaflet free edges separation. Yet statistical correlation was found between the maximum stitch tension and maximum values of all mentioned geometrical dimensions. The marked dependency of stitch tension on the septo-lateral dimension of the annulus was clearly confirmed by a subsequent animal study of the same group. 77 These results suggested that in presence of progressive annular dilation the durability of the edge-to-edge technique may be reduced by excessive stitch tension and leaflets stresses, and support the need for concomitant annuloplasty in case of annular dilation, as confirmed by subsequent clinical outcomes reported in Ref. 50

A series in vitro analyses on the determinants of stitch tension have been conducted on excised porcine valves through the GeorgiaTech Left Heart Simulator (see "Effects on MV Geometry and Hemodynamics" section). In Jimenez et al. 32 valves were tested in variable annular conditions (baseline, dilated and contracted, corresponding to 6.86, 8.22, and $5.50 \mathrm{~cm}^{2}$, respectively), with and without edge-to-edge central suture. In the latter case, the suture was instrumented with a $\mathrm{C}$-shaped miniature transducer, positioned on the ventricular side of the leaflets so to 
minimizing interference with leaflets coaptation. Two kinds of experiments were conducted: using steady-state flow conditions at variable diastolic flow rate values (from 0 to $30 \mathrm{~L} / \mathrm{min}$ ), with the aim to assess possible relationships between flow rate and stitch tension, and in physiologic pulsatile conditions, with peak transmitral pressure of 100,120, and $140 \mathrm{mmHg}$ and cardiac output of 4,5 , and $6 \mathrm{~L} / \mathrm{min}$, so to measure the timecourse of the stitch tension and assess possible correlations with geometrical and hemodynamic measurements. In the steady-state experiments, notable decrease in effective orifice area was measured in presence of edge-to-edge suture as compared to pre-repair conditions. The stitch tension increased more than linearly with trans-mitral flow rate; annular dilation decreased stitch tension, while annular contraction increased it. Pulsatile experiments showed that annular dilation caused MR even in presence of edge-to-edge suture. Differently from in vivo findings in ovine models, a bi-modal time-course of stitch tension was measured, with a systolic peak pairing the peak in trans-mitral pressure and a diastolic one pairing the peak in trans-mitral flow rate. The ratio between the two peak values depended on annular size: with baseline and contracted annulus, diastolic peak was higher $(0.072 \pm 0.021$ and $0.071 \pm 0.020 \mathrm{~N}$, respectively); with dilated annulus, the systolic peak was higher $(0.092 \pm 0.030 \mathrm{~N})$. Also, multivariate ANOVA analyses showed that transmitral pressure and flow rate, as well as annular area, had a significant effect on stitch tension. These data further highlighted the need to complement the edge-to-edge repair with annuloplasty in case of annular dilation. Also, the bi-modal stitch tension time-course suggested that after edge-to-edge repair leaflets tissue next to the stitch is loaded with a doubled frequency as compared to physiological conditions, further enhancing the need for keeping stitch tension as low as possible. This concept was confirmed by He and colleagues 28 in a separate series of in vitro tests on excised porcine valves corrected through central edge-toedge suture; in this further study, a $4 \times 4$ array of miniaturized markers was placed on the ventricular side of the anterior leaflet belly. Pulsatile tests were conducted in physiological hemodynamic conditions $(5 \mathrm{~L} / \mathrm{min}$ flow rate, $120 \mathrm{mmHg}$ peak systolic transmitral pressure), and markers position was acquired with two high speed digital cameras on two almost perpendicular planes. Acquired positions were than reconstructed in the 3D space and interpolated with biquadratic finite element for the computation of strains. These were used as an indirect estimation of leaflets stresses induced by the surgical repair, and showed both a systolic and a diastolic 
peak.

This analysis was expanded recently 60 ; pulsatile experiments were conducted on excised porcine valves before and after selective cutting of marginal chordae tendineae, so to test the effects of P2 flail, which characterizes a relevant fraction of degenerative MR cases. Valves were tested after annuloplasty with a rigid and complete prosthetic ring of two sizes. As for the previous study, central edge-to-edge suture was performed and instrumented. Before chordae cutting, valves were continent, and MR occurred after flail was obtained. Both before and after chordae cutting, time-dependent stitch tension showed a systolic and a diastolic peak; in particular, the diastolic tension peak increased with the size imposed to the annulus, and showed no statistically significant difference tests with intact and cut chordae. Also, its magnitude was not correlated to flail extent nor width. These findings suggested that the presence of P2 flail should not limit the durability of the edge-to-edge repair.

The detailed analysis of the space-distribution of leaflets stress associated to edge-to-edge correction was carried out through different finite element models. Although developed over an entire decade since $2002,14,44,79,81$ and despite the increasing possibility of including detailed and realistic MV geometry and boundary conditions into finite element models, 38 these all adopted idealized MV geometrical models based on limiting simplifying assumptions: MV symmetry with respect to the plane passing through the septo-lateral diameter of the valve and normal to the valvular orifice, planar and static annulus, static PMs, simplified leaflets profile. Also, none of these models accounted for the pathological features that characterize MR. The most notable difference between these models regards the constitutive modelling of leaflets mechanical properties, which are assumed linear and transversely isotropic in Ref. 79, non-linear and isotropic in Ref. 14, and non-linear and anisotropic in Ref. 44

The first finite element analysis of this kind was proposed by our research group. 79 In that model, leaflets dimensions derived from ex vivo measurements on porcine valves and adopted in previous studies 7 were used, and a circular annulus was used, this geometry being intended to mimic diastolic annular geometry rather than systolic one. Also, the model included only marginal chordae. Despite these limitations, the model 
provided the first quantification of leaflets stresses following edge-to-edge repair, and of the influence of suture dimensions, annular dilation, and peak diastolic trans-mitral pressure. Diastolic peak von mises stresses resulted comparable to those computed at peak systole, again suggesting the doubled frequency of leaflets mechanical loading as compared to physiological conditions. Independently from other parameters, as the suture extent decreased by $50 \%$, radial stresses (i.e., normal stresses directed from the annulus to the free edge) decreased both close to the suture and close to the annulus, while circumferential stresses increased next to the suture and decreased in the annular region. Annular dilation, simulated by a $20 \%$ increase in annular diameter, led to massive stress increase regardless of other parameters, appearing the most critical factor for the durability of the repair from a mechanical standpoint. These main findings were confirmed by later studies, 14,44 although the precise value of computed stresses changed owing to the different constitutive laws adopted in the different models.

The influence of suture position and extension was more thoroughly analyzed by Dal Pan and colleagues. 14 In their model, they adopted a fixed and D-shaped annulus, which better mimics the 2D profile of the real valvular sub-structure, and accounted for leaflets non-linear stress-strain behavior, but neglected leaflets anisotropy and, based on a set of preliminary simulations, simulated the effect of chordae tendineae simply through a kinematic restraint imposed to leaflets free edge. The model was used to simulate the effect of 6 different extents of the suture $(1-6 \mathrm{~mm}$, step $1 \mathrm{~mm}$ ) in the central positions, seven different positions of a $1 \mathrm{~mm}$ suture (distance from the free edge mid-point equal to 0-18 $\mathrm{mm}$, step $3 \mathrm{~mm}$ ), and seven different annular sizes ( -16 to $+32 \%$ with respect to baseline conditions, step $8 \%$ ). For each configuration, the authors related stresses to trans-mitral pressure and flow rate making use of the relationship reported in Ref. 48 between these two parameters and identifying the operating point of the valve. Computed results confirmed the indications provided by Votta et al.,79 moreover showed that the potentially critical points of the suture in terms of stress transfer to the leaflets are at the suture ends. According to simulations, a lateral shifting of the suture implies better MV functionality in terms of trans-mitral pressure, leaflets mobility, and leaflets stresses. An increase in suture extent induced lower stresses, but reduced leaflets mobility, while annular dilation increased stresses and reduced leaflets mobility. 


\section{Possible Connections Between Post-repair Biomechanics and Repair Durability}

The motivation underlying the analysis of the biomechanical effects of surgical interventions in general, and of the edge-to-edge technique in particular, consists in the effect of fluid flow forces and valve strains/stresses on valvular cells homeostasis and extra-cellular matrix (ECM) deposition/disruption. In fact, the durability of surgical repairs depends on the preservation of valve tissue integrity, as well as on the absence of abnormal remodeling that could alter valvular function.

As regards fluid flow forces, in a recent in vitro study Tan and colleagues showed that shear stresses generated on embryonic atrioventricular valve tissue by pulsatile fluid flow led to the upregulation of fibrous ECM expression at transcript and protein level, as compared to no flow conditions. This evidence suggests a role of fluid-dynamic forces in the early development of atrioventricular valves, 72 and it is hence reasonable that the same mechanism may influence the remodeling of already developed valves. Also, Gould and colleagues reported that wall shear stresses generated by fluid flow regulate affect the endothelial cells covering the aortic side of aortic valve leaflets. In particular, the pathological decrease of wall shear stresses and disturbed flow conditions can lead to endothelial cell apoptosis, inducing non-specific permeability of the valvular tissue, and tissue inflammation secondary to the abnormal recruitment of inflammatory cells and cytokines. 25 This mechanism may take place also in the tissue of mitral leaflets, and in particular on their ventricular side, in case of abnormal LV fluid-dynamics associated to pathologies or surgical repair.

As regards structural strains and stresses, Pham and Sun, 59 based on biaxial mechanical testing performed on aged human mitral leaflets from normotensive and hypertensive subjects, showed that in the leaflets from elderly subjects calcifications are present next to chordae tendineae insertions, as well as in the annular region of the anterior leaflet. Finite element studies from the literature frequently indicate these locations as sites of high stresses (e.g., Ref. 34), and hence it can be speculated that local high values in cyclic stresses induced by pulsatile pressure drive progressive calcification. Moreover, in the same study, it was shown that mitral leaflet tissue from hypertensive subjects was less extensible and 
stiffer as compared to mitral leaflet tissue from normotensive subjects, thus suggesting a role of pressure, and hence of mechanical stresses, in the remodeling of ECM microstructure.

Moreover, heart valve leaflets, including MV leaflets, contain valve interstitial cells (VICs), which play a role in regulating ECM deposition. 43 Different studies focused on the aortic valve have shown that strains at the valve tissue level are transferred to VICs, influencing their differentiation and activity (e.g., Refs. 25, 31, 74).

Despite the existence of the mentioned evidences of correlation between valve biomechanics and valve tissue damage/remodeling, a sound quantification of the threshold values of strains, stresses and wall shear stresses that may trigger these phenomena is not available. This lack of information limits the possibility to use computational models to infer about the long-term effects of MV surgical repair in absolute terms, since the computed values not only are influenced by unavoidable assumptions underlying the models, but also cannot be compared to precise terms of comparison. As a consequence, currently computational studies compare biomechanical variables computed in the simulation of pathologies or surgical treatments to those computed when mimicking physiologic conditions, and speculations are made under the hypothesis that notably altered levels of wall shear stress/stress/strain can lead to pathologic tissue remodeling or to tissue failure. This limitation should be taken into account whenever interpreting the results of computational biomechanical models.

\section{Structural and Functional Effects of the Mitraclip Procedure ${ }^{\circledR}$ : A Novel Finite Element Analysis}

As previously mentioned, the latest progress in the story of the edge-toedge technique is the transition from the surgical to the percutaneous access, which is currently performed in the real world through the Mitraclip ${ }^{\circledR}$ system. Here we propose a novel analysis of the biomechanical effects of the latter, based on image-based finite element modeling, which addresses several of the limitations present in the only study available in the literature that quantified MV biomechanics in this specific scenario. 7 That study is based on the same idealized geometrical model and boundary conditions adopted in Ref. 14 and does not account for any MV pathological feature; these simplifications do not allow for computing 
biomechanical variables of clinical interest with reference to a real world clinical scenario.

\section{Materials and Methods}

\section{Cardiac Magnetic Resonance Imaging}

Three patients scheduled for surgical repair of posterior prolapse due to fibroelastic deficiency (FED) were considered in the study at single University Hospital. Although not undergoing Mitraclip ${ }^{\circledR}$ implantation, they well resembled the MV functional and morphological conditions often characterizing real candidates: all were affected by chordal rupture or elongation, and the morphology of their MVs was consistent with the criteria of the EVEREST clinical trial (Table 1). Pre-operative CMR cine sequences were acquired with a 3.0 T scanner (TX Achieva, Philips Medical System, Eindhoven, The Netherlands) on 18 cut-planes evenly rotated around the axis ideally passing through the annular center and aligned with the LV long-axis (Fig. $3 \mathrm{a}$ ). In-plane spatial resolution and slice thickness were equal to 0.78 and $8 \mathrm{~mm}$, respectively. 30 frames/cardiac cycle were acquired with R-wave triggering.

\section{Table 1}

General characteristics of the enrolled patients

\begin{tabular}{|c|c|c|c|}
\hline $\begin{array}{l}\text { Subject } \\
\text { ID }\end{array}$ & Patient 1 & Patient 2 & Patient 3 \\
\hline Gender & M & M & $\mathrm{F}$ \\
\hline $\begin{array}{l}\text { Age } \\
\text { (years) }\end{array}$ & 57 & 57 & 80 \\
\hline $\begin{array}{l}\text { Weight } \\
(\mathrm{kg})\end{array}$ & 66 & 90 & 65 \\
\hline $\begin{array}{l}\text { Height } \\
(\mathrm{cm})\end{array}$ & 166 & 178 & 162 \\
\hline $\mathrm{BSA}\left(\mathrm{m}^{2}\right)$ & 1.73 & 2.08 & 1.69 \\
\hline $\begin{array}{l}\text { Heart } \\
\text { Rate } \\
\text { (bpm) }\end{array}$ & 57 & 81 & 62 \\
\hline $\begin{array}{l}\mathrm{A} 2 \mathrm{D} / \mathrm{BSA} \\
\left(\mathrm{cm}^{2} / \mathrm{m}^{2}\right)\end{array}$ & 7.71 & 8.68 & 8.78 \\
\hline$D_{\mathrm{SL}}(\mathrm{mm})$ & 38.7 & 40.8 & 40.6 \\
\hline
\end{tabular}




\begin{tabular}{|l|l|l|l|}
\hline $\begin{array}{l}D_{\mathrm{CC}}(\mathrm{mm}) \\
e(-)\end{array}$ & 44.3 & 53.1 & 46.5 \\
\hline $\begin{array}{l}\text { IPP } \\
\text { region }\end{array}$ & P2 & 1.30 & 1.14 \\
\hline $\begin{array}{l}\text { Chordae } \\
\text { anomaly }\end{array}$ & $\begin{array}{l}\text { Double secondary } \\
\text { chordal elongation, } \\
\text { from both PMs to P2 }\end{array}$ & $\begin{array}{l}\text { Triple primary } \\
\text { chordal rupture, } \\
\text { from both PMs to }\end{array}$ & $\begin{array}{l}\text { P2 } \\
\text { Pingle primary } \\
\text { chordal rupture, } \\
\text { from APM to P2 }\end{array}$ \\
$\begin{array}{l}\text { BSA: body surface area; A2D: annular area projection on MV plane; DSL: } \\
\text { septo-later diameter; DCC: commissural diameter; } e \text { : eccentricity (DCC/DSL; } \\
\text { IPP region: main prolapsing scallop in the posterior MV leaflet }\end{array}$ \\
\hline
\end{tabular}

\section{Figure 3}

Reconstruction of MV 3D models from CMR images. (a) Subset of the 18 long axis CMR planes acquired at each time point; $\mathrm{A}$ and $\mathrm{B}$ indicate the center of the mitral orifice and the ventricular apex, and define the rotation axis for the sequence. (b) Single CMR plane and traced MV sub-structures: annular points (yellow dots), leaflets profile (green crosses), papillary muscle tips (cyan dots). The orange dashed line indicates the rotation axis of the sequence. (c) Leaflets profile manually traced on CMR planes (black dots) and approximated by 3D Fourier functions (dashed red lines). (d) 3D point cloud obtained by sampling the Fourier functions approximating leaflets profile. (e) Leaflets triangulated mesh; the color code indicates nodes' $z$-coordinate. (f) Complete model of the MV, including chordae tendineae. Red dots at their lower ends indicate chordae origins from papillary muscles tips 


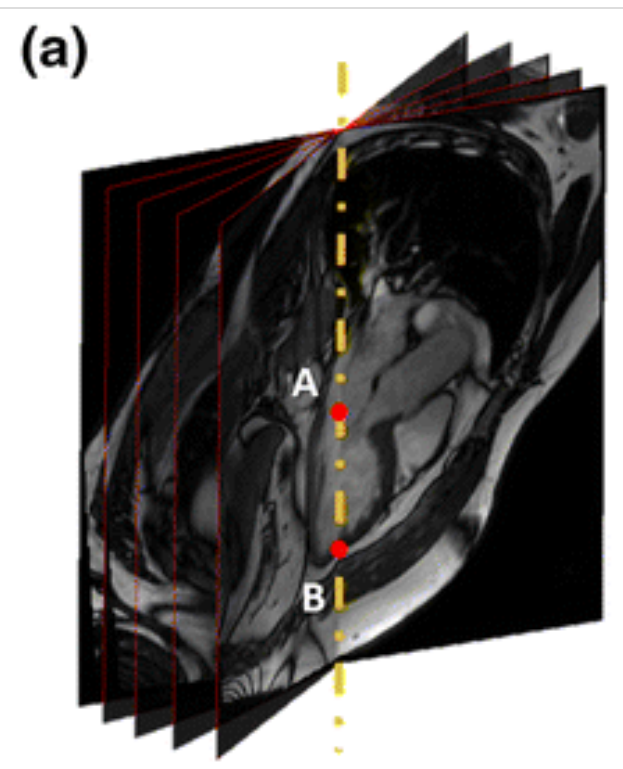

(d)

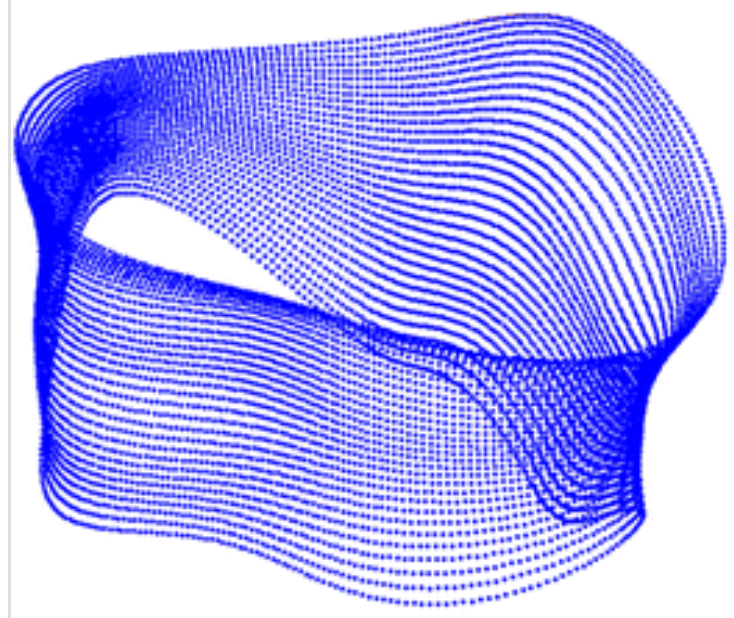

(b)

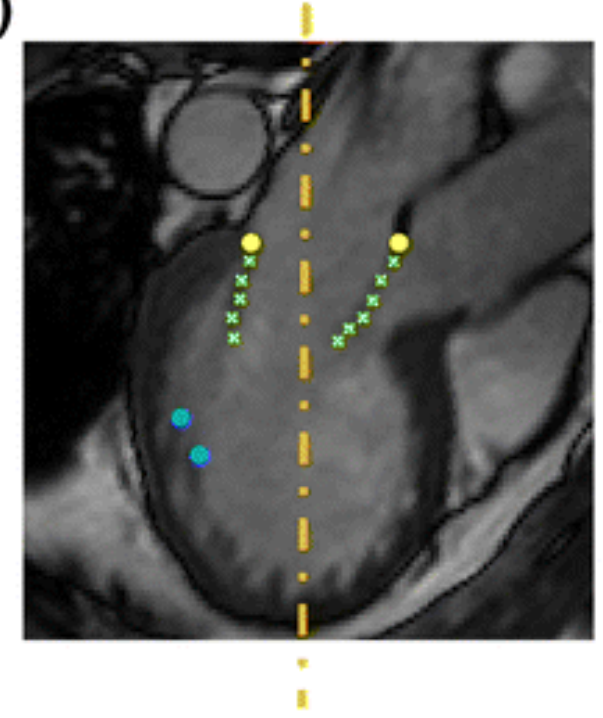

(e)

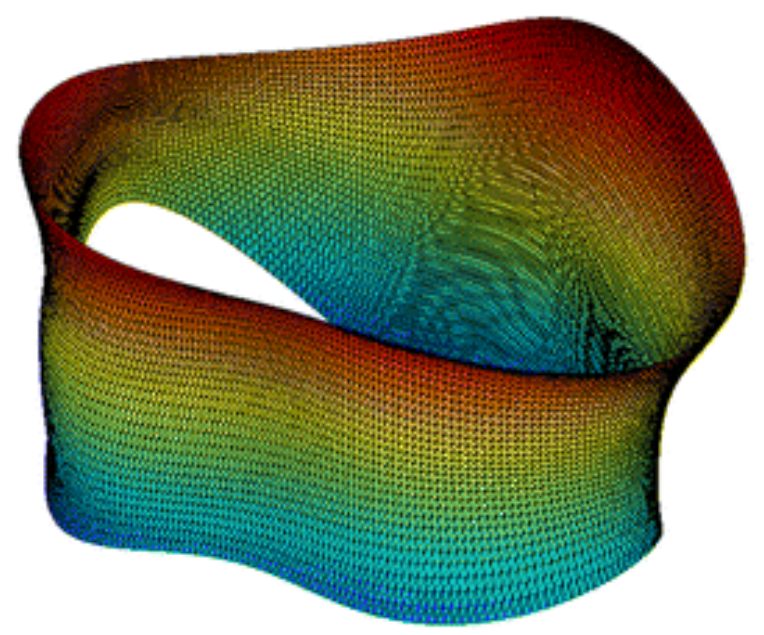

(f)

(c)
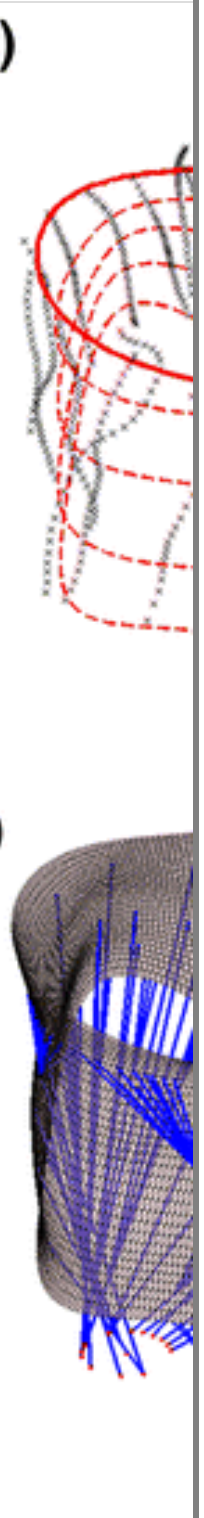

\section{Image Processing and MV 3D Geometrical Model}

Software developed in MATLAB (The MathWorks Inc., Natick, MA, United States) was used for (i) the manual tracing of MV substructures on CMR images, (ii) the automated reconstruction of MV 3D models, and (iii) the automated generation of the input files for subsequent finite element analyses, which were performed through ABAQUS/Explicit 6-10 (SIMULIA, Dassault Systèmes).

Regarding manual tracing, for every time-point and for every cut-plane, two annular points were identified, and PMs were traced whenever visible (Fig. 3 b). For the time-points corresponding to end-diastole (ED) and peak systole (PS), on each cut-plane multiple points were selected on the MV leaflets profile; for each leaflet, traced points were automatically 
interpolated with cubic splines, which were sampled in 32 points running from the annulus to the free margin and evenly distributed along the $z$-axis (Fig. 3 b). Also, when visible, a point located within the aortic root was selected.

As for previous studies, 68 the 3D geometrical model of the MV was reconstructed at ED, when the MV was hypothesized approximately unloaded. In order to realistically capture MV leaflets surface while avoiding artifacts due to manual tracing, the following procedure was adopted:

- a cylindrical reference frame was set with the origin in the center of mass of the points on MV leaflets free edge, and the $z$-axis normal to the least-square plane of the annular points;

- in this local reference frame, the radial $(\rho)$ and axial $(h)$ positions of the $i$ th point of every leaflet spline were then approximated through 4 th order Fourier functions of the angular position $(\vartheta)$ in the form:

$$
\left\{\begin{array}{l}
\rho_{i}(\vartheta)=a_{0}^{i}+\sum_{k=1}^{4}\left[a_{k}^{i} \cdot \sin (k \vartheta)+b_{k}^{i} \cdot \cos (k \vartheta)\right] \\
h_{i}(\vartheta)=c_{0}^{i}+\sum_{k=1}^{4}\left[c_{k}^{i} \cdot \sin (k \vartheta)+d_{k}^{i} \cdot \cos (k \vartheta)\right]
\end{array} i=1, \ldots, 32,\right.
$$

where $a_{k}^{i}, b_{k}^{i}, c_{k}^{i}, d_{k}^{i}$ are the coefficient of the $i$ th Fourier functions (Fig. $3 \mathrm{c}$ );

- the Fourier function $h_{1}(\vartheta)$, which approximated the axial position of the annular points, was used to identify four reference points: the saddle horn $(\mathrm{SH})$, the midpoint of the posterior annulus (P2), and the two commissures $\left(C_{1}\right.$ and $\left.C_{2}\right)$. SH and $\mathrm{P} 2$ were identified as the two local maxima of $h_{1}(\vartheta)$, located next to the aortic root and in the opposite position, respectively. $C_{1}$ and $C_{2}$ were identified as the two local minima of $h_{1}(\vartheta)$. These four reference points identified four tracts on the annulus, which were sampled uniformly, for a total number of points that varied between 200 and 300 from patient to patient, depending on the size of the annulus. The same sampling was performed on every Fourier function;

- sampled points were connected into a mapped mesh of 3-node shell 
elements (ABAQUS S3 shell element type);

- a regionally varying thickness distribution was assigned to the leaflets, consistently with the one proposed in Ref. 40, with a maximum thickness around the fibrous trigones $(1.69 \mathrm{~mm})$, and an average value of $1.32 \mathrm{~mm}$ on the anterior leaflet and $1.26 \mathrm{~mm}$ on the anterior and posterior leaflets one, respectively;

- the 3D model was completed by PM tips and chordae tendineae, modeled as in a previous work. 68 For every each PM, a point cloud was obtained from the selection made on the different cut-planes; a circumference of $3 \mathrm{~mm}$ radius was centered in the center of mass of the point cloud and oriented parallel to the valvular plane, (i.e., the least square plane approximating the annular profile). Thirteen points were defined on each circumference as the origins of chordae tendineae. Chordae tendineae of three orders, i.e., first order or marginal, second order or basal, and strut chordae, were modeled as truss elements (ABAQUS T3D2 element type); their number, the corresponding branched structure, the origin from PMs and insertion sites on the leaflets were defined in accordance to ex vivo findings 42 and indications by clinicians. In order to reproduce posterior leaflet prolapse or flail as visible from CMR images, chordae tendineae were locally elongated or ruptured, accordingly with intraoperative evidence reported by surgeons (Table 1 ).

\section{Tissues Mechanical Properties}

MV leaflets mechanical response is non-linear and anisotropic, owing to the presence of collagen fibers dispersed within the tissue, which, although not perfectly aligned, are preferentially oriented along a main direction, making the tissue mechanical response in this direction stiffer than in the transversal one. The resulting stress-strain behavior was described through the constitutive model recently proposed in Ref. 45 , and implemented in a VUMAT subroutine:

$$
\psi\left(I_{1}, I_{4}\right)=C_{10}\left(I_{1}-3\right)+\frac{c_{0}}{2}\left[(1-\delta) e^{c_{1}\left(I_{1}-3\right)^{2}}+\delta e^{c_{2}\left(I_{4}-1\right)^{2}}-1\right]
$$

where $\psi$ is the energy density function, $I_{1}=\operatorname{trace}(\mathbf{C})$ and $I_{4}=\mathbf{N C N}$ are the first and fourth invariants of the right Cauchy-Green strain tensor 
$\mathbf{C}, \mathbf{N}$ being the versor identifying collagen fibers direction in the unloaded configuration; with reference to the same cylindrical reference frame adopted for the reconstruction of leaflets $3 \mathrm{D}$ model, $\mathbf{N}$ was assumed running in the circumferential direction. $\delta$ is a parameter ranging from 0 to 1 and governing the level of material anisotropy, hence accounting for possible different degrees of dispersion if collagen fiber direction. $c_{0}, c_{1}, c_{2}$ are the remaining constitutive parameters. All of the constitutive parameters were identified by fitting data reported in the literature and based on ex vivo biaxial mechanical testing of porcine MV leaflets 54 (Table 2).

\section{Table 2}

Constitutive parameters of leaflets tissue

\begin{tabular}{|l|l|l|l|l|l|}
\hline & $\boldsymbol{C}_{\mathbf{1 0}}(\mathbf{k P a})$ & $\boldsymbol{c}_{\mathbf{0}} \mathbf{( k P a )}$ & $\boldsymbol{c}_{\mathbf{2}}$ & \multicolumn{1}{|c|}{$\boldsymbol{c}_{\mathbf{2}}$} & $\boldsymbol{\delta}$ \\
\hline Anterior leaflet & 0.128 & 0.018 & 28.661 & 38.579 & 0.088 \\
\hline Posterior leaflet & 0.248 & 0.145 & 10.150 & 17.973 & 0.044 \\
\hline
\end{tabular}

Chordae tendineae response was assumed isotropic and non-linear, and was modelled through hyperelastic strain energy functions available in the ABAQUS material library: a 2nd order polynomial model for first order and strut chordae, and a 5 th order Ogden model for 2 nd order chordae. The corresponding constitutive parameters were defined by fitting uniaxial test data obtained from literature. 37 Constant cross-sectional area values of $0.40,1.15$, and $0.79 \mathrm{~mm}^{2}$ were assigned to first order, strut and second order chordae, respectively. Chordae tendineae were locally removed or elongated to reproduce the chordae pathology affecting each patient (Table 1).

The density assumed for all valvular tissues was equal to $10.4 \mathrm{~g} / \mathrm{cm} 3$, a value ten times higher than the real one, to take into account the inertial effects of the blood volume spanned and moved by MV leaflets during closure. Such volume is approximately ten times larger than MV leaflets volume.

\section{Boundary Conditions}

The motion of annulus MA and PMs was derived from CMR and modeled 
as nodal displacements.

As previously mentioned, for every time-point of the cine-CMR sequence annular points were manually traced and their 3D position was approximated through Fourier functions. $\mathrm{SH}, \mathrm{P} 2, C 1$ and $C 2$ were identified as the peaks and the minima of these functions, so that four annular tracts could be identified with their respective ends. Each of the four annular tract was uniformly sampled into the same number of points adopted at ED, and the corresponding 3D displacements could be computed.

As regards PMs, for each time-point the centers of mass of the corresponding point-clouds were computed, and their time-dependent 3D displacements with respect to their position at ED was imposed.

A physiological time-dependent transvalvular pressure drop ranging between -5 and $110 \mathrm{mmHg}$ was applied on the ventricular side of the leaflets (Fig. $4 \mathrm{a})$.

\section{Figure 4}

Simulations setup. (a) Time-dependent pressure load applied to MV leaflets when simulating the whole cardiac cycle. (b) Complete 3D model of the MV used for the pathological simulation; red dots highlight the nodes on the annulus and on the PMs tips, whose motion is imposed via time-dependent nodal displacements when simulating the whole cardiac cycle. (c) 3D model of MV leaflets and Mitraclip ${ }^{\circledR}$ arms (chordae tendineae are not depicted for the sake of clarity) used for the Mitraclip ${ }^{\circledR}$ two-step simulation. In step 1, Mitraclip ${ }^{\circledR}$ arms initially next to the mid-point of the respective leaflet free edge, and are pulled side by side by rigid rotation and shortening of a first set of connector element (red lines). In step 2, the two arms are kept together by a second set of connector element (red lines), while the time-dependent pressure and displacements adopted in B) are applied 


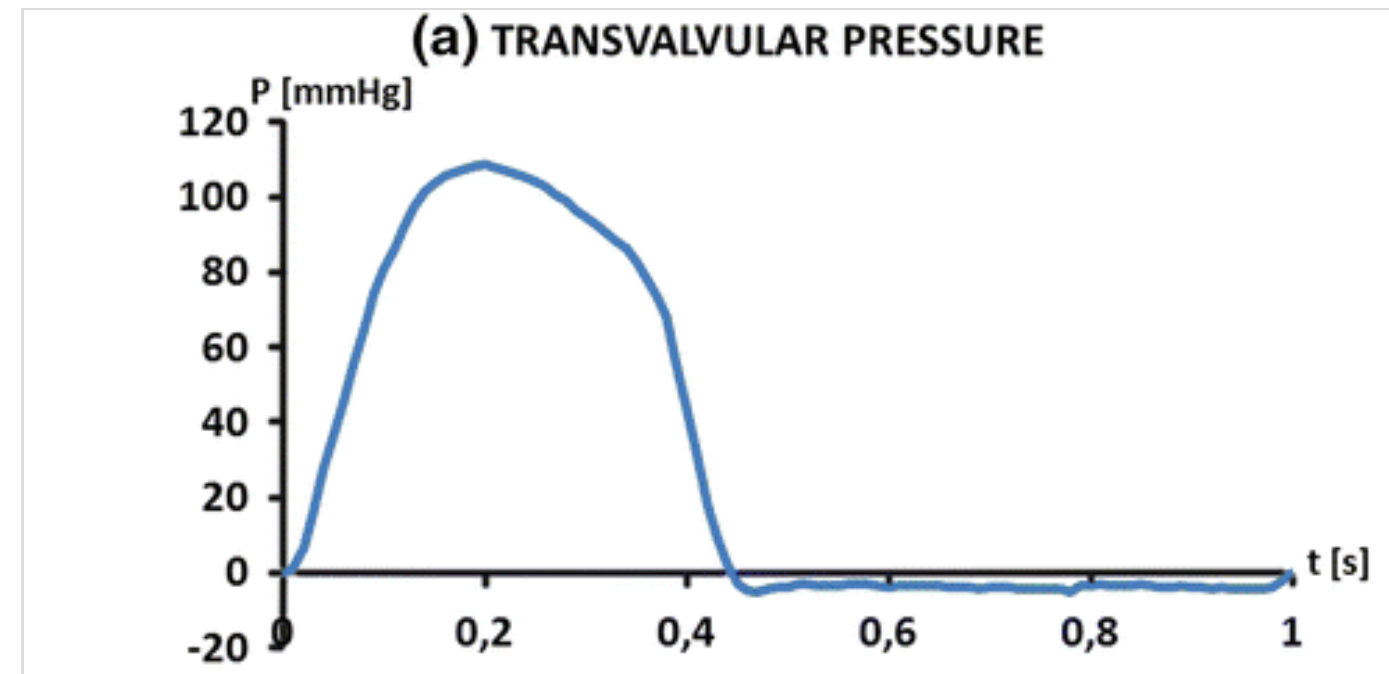

(b) PATHOLOGICAL SIMULATION

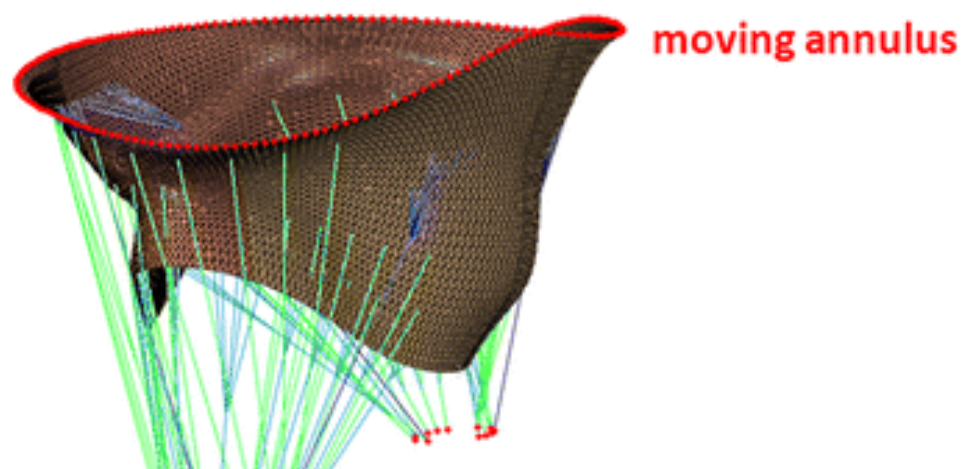

moving PMs

(c) MitraClip ${ }^{\otimes}$ SIMULATION

STEP 1

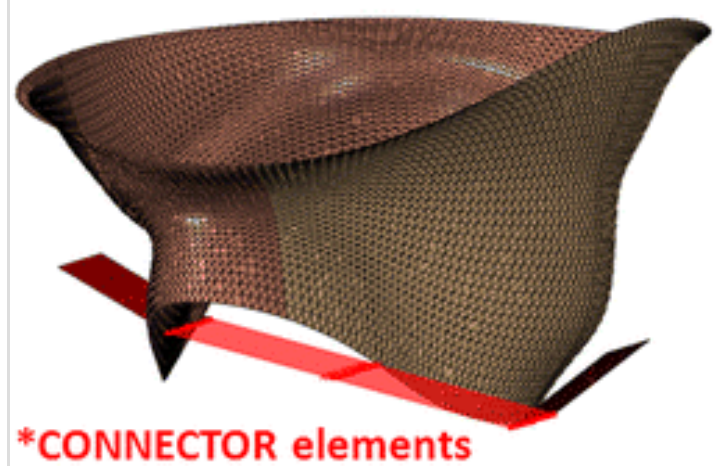

to be shortened
STEP 2

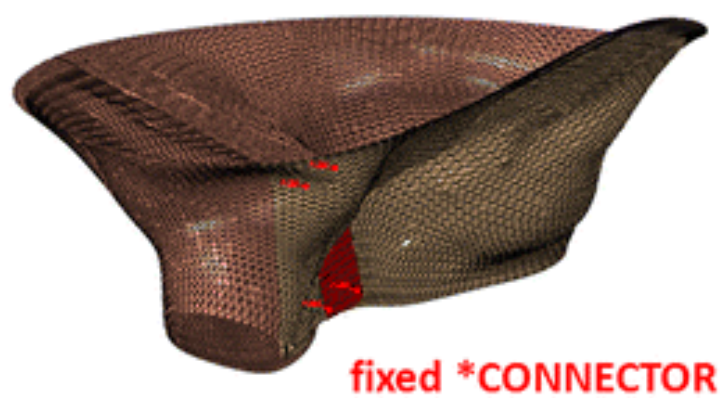

elements

\section{Contacts}

Contact was modeled for the atrial side of MV leaflets, and between the ventricular side of MV leaflets and the two Mitraclip ${ }^{\circledR}$ arms. The hard contact model was adopted to describe the contact pressure-overclosure behavior between surfaces. When modeling contact between MV leaflets, a friction coefficient of 0.05 was adopted, as in previously published 
studies. 67 Contact between the atrial side of MV leaflets and the two Mitraclip ${ }^{\circledR}$ arms was assumed rough, i.e., with no possible slippage, and with no separation after contact was achieved.

\section{Mitraclip ${ }^{\circledR}$ Model}

Under the assumption that optimal gripping is achieved, the two arms of the Mitraclip ${ }^{\circledR}$ device were modeled as two perfectly rigid rectangular plates, $5 \mathrm{~mm}$ wide and $7 \mathrm{~mm}$ long. $1 \mathrm{In}$ order to set the initial position of the plates, for each patient the site of the valvular defect was identified as the position of the node of the posterior leaflet free edge that was maximally everted towards the atrium. The two plates were then positioned so to have their centerline aligned with the identified node in a septolateral view. They two plates were connected by two sets of ABAQUS axial connector elements, which do not model an actual component of the device, but rather allow for imposing mutual kinematic constraints between the nodes of the two plates located at the ends of each connector element (see next subsection for details). The first set connects 1-by-1 the nodes of the lower and of the arms, the second set consists of four connector elements connecting the four corners of the arms. The initial positioning of the two arms in the 3D space, and the boundary conditions needed to drive their displacement, were automatically generated based on the choice of the reference node on the posterior leaflet.

\section{Simulations Set-Up}

For each patient, two simulations were run to assess MV biomechanics in pathological conditions (patho-sim) and following Mitraclip ${ }^{\circledR}$ implantation (mc-sim).

In the patho-sim, a full cardiac cycle was simulated in a single step, with no Mitraclip ${ }^{\circledR}$ device. Time-dependent kinematic and loading boundary conditions were applied to the corresponding MV substructures, while leaflets contact was activated (Fig. 4b).

In the mc-sim, two steps were implemented: in the first one, the arms of the Mitraclip ${ }^{\circledR}$ device were tilted, so to become vertical and mutually parallel, while the connector elements at the lower end of the two arms were shortened until a gap of $1.4 \mathrm{~mm}$ was left between the arms. 1 Contextually, leaflets were grasped by the arms owing to the contact 
between them and the ventricular side of MV (Fig. 4c); of note, the plates representing the clip remained mutually parallel, but could rotate/tilt following the interaction with leaflet tissue. In the second step, the length of the connector elements linking the four corners of the Mitraclip ${ }^{\circledR}$ arms was kept fixed, so to prevent relative displacements between them, and a full cardiac cycle was simulated, applying the same time-dependent boundary conditions applied in the patho-sim (Fig. 4c).

\section{Results}

In each simulated condition (i.e., pre-operative and following Mitraclip ${ }^{\circledR}$ implantation), systolic function was assessed in terms of leaflet mechanical stresses and systolic coaptation area (CoA), i.e., the extent of the region where the anterior and posterior leaflets overlap after MV closure. Since Mitraclip ${ }^{\circledR}$ implantation is subject to the same potentially drawbacks in diastole, diastolic function was evaluated: diastolic stresses were computed and the diastolic orifice area was quantified in $2 \mathrm{D}$, i.e., considering the projection of leaflets free edge onto the least-square plane of the annulus.

\section{Semi-quantitative Comparison of the Model Vs. Imaging Data}

In order to assess the reliability of our modeling strategy at least from a semi-quantitative standpoint, for the pre-operative simulations the computed systolic leaflets configuration was compared vs. ground truth data from cardiac MRI. In particular, for each modeled MV the CMR tomographic plane that most clearly showed the valvular defect was chosen (Figs. $5 \mathrm{a}, 5 \mathrm{e}$, and $5 \mathrm{i}$ ), and rigidly roto-translated through the same coordinate transformation initially applied to the 3D geometrical model of the MV. It was then used to identify the corresponding cross section on the FEM. The leaflet elements crossed roto-translated CMR plane were selected, and the corresponding nodes were projected onto the crosssectional plane; projections were then mapped back on the original CMR plane and interpolated through a piecewise linear line. The spline interpolating the leaflet point manually traced on the selected CMR plane at peak systole (Figs. $5 \mathrm{~b}, 5 \mathrm{f}$, and $5 \mathrm{j}$ ) and the corresponding leaflet profile obtained through the sectioning of the FEM (Figs. $5 \mathrm{c}, 5 \mathrm{~g}$, and $5 \mathrm{k}$ ) were visually compared. Some discrepancies emerged, which mainly affected the anterior leaflet: in patients 1 and 3, the computed profile of the anterior leaflet was excessively convex towards the ventricle in the belly/annular 
region, while in patient 2 the free margin of the anterior leaflet was displaced towards the ventricular apex. However, the configuration of the posterior leaflet was really well captured in terms of shape, and bulging (patients 1 and 3) or flail (patient 2). Thus, the key aspect of the simulated pathological condition was well captured in all of the three MVs.

\section{Figure 5}

Computed systolic configuration vs. respective ground truth data. For each patient, at peak systole a CMR cut-plane is shown, and the region of interest for the MV is framed by a white square (panels a, e, and i). The region of interest for the MV is zoomed and MV leaflets profile, as visible from CMR images, interpolated via cubic splines is highlighted (panels $b, f$, and $\mathrm{j}$ ). After FEM simulation of MitraClip ${ }^{\circledR}$ implantation, the same cut-plane is shown; the computed leaflets cross section on the CMR plane (panels c, g, and k) and 3D configuration (panels $\mathrm{d}, \mathrm{h}, \mathrm{l}$ ) are superimposed to the clinical image for semi-quantitative comparison 

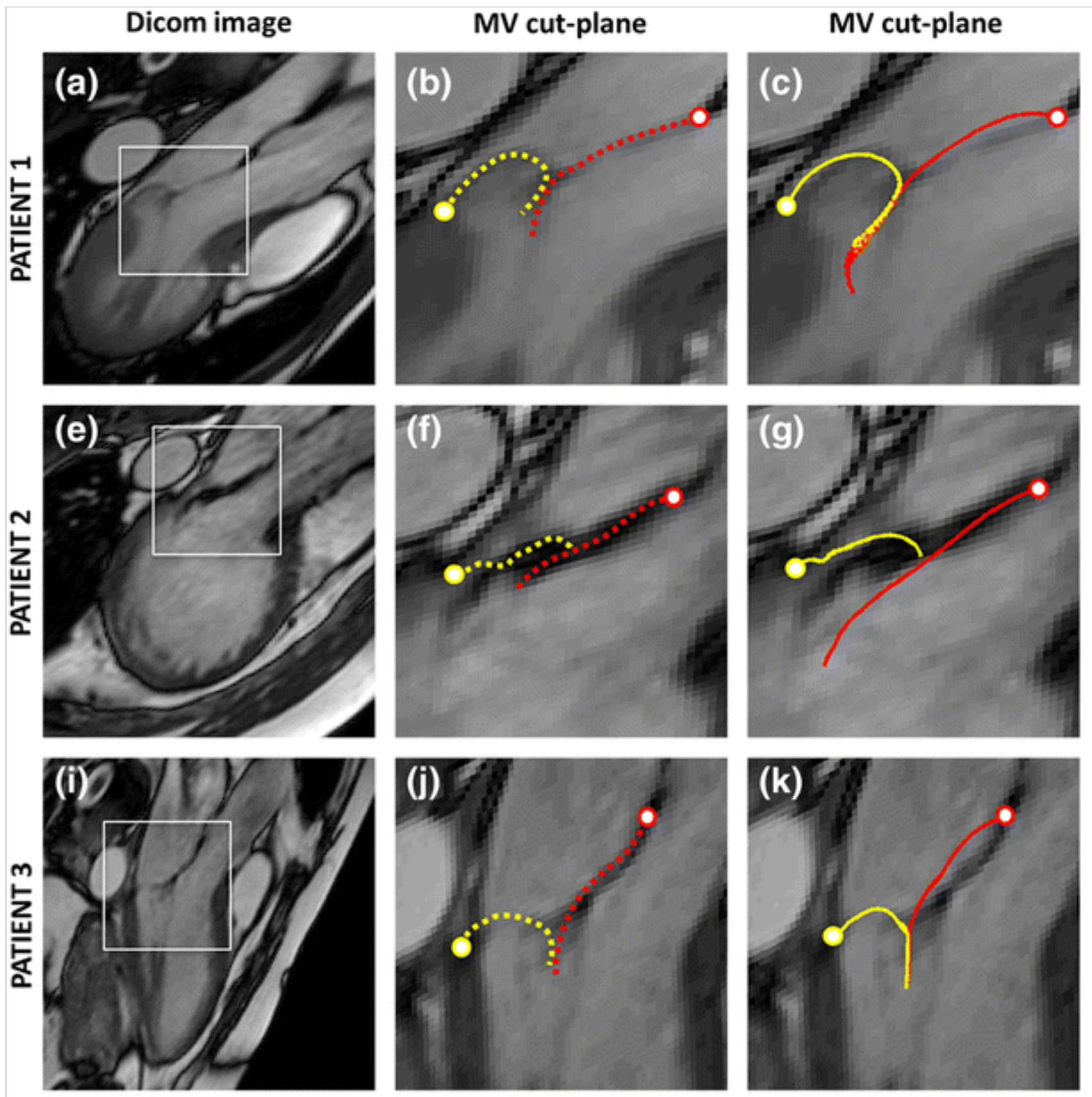

CMR Dicom
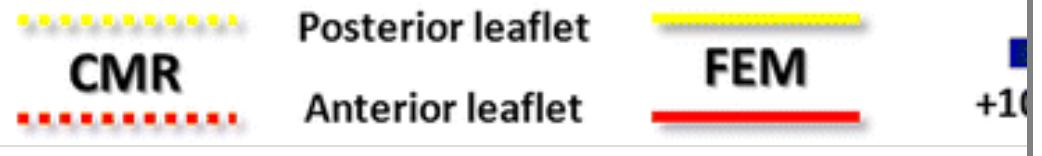

\section{Systolic Coaptation Area}

Coaptation area (CoA) at peak systole was computed in both preoperative conditions and after Mitraclip ${ }^{\circledR}$ procedure (Fig. 6). MitraClip ${ }^{\circledR}$ implantation increased CoA by $11-40 \%$ : the largest recovery was computed in patient $2(+39.8 \%)$ who reported, in preoperative conditions, an irrelevant level of coaptation along the mid-part of the posterior leaflet. For patients 1 and 3, characterized by less relevant lack of coaptation in pre-operative conditions, a lower but still notable CoA recovery was computed (11.5 and $15.1 \%$, respectively).

\section{Figure 6}


Leaflets maximum principal stresses at peak systole in pathological conditions (left) and following Mitraclip ${ }^{\circledR}$ implantation (right). For each patient, the atrial view and a $3 \mathrm{D}$ view of the valve are provided

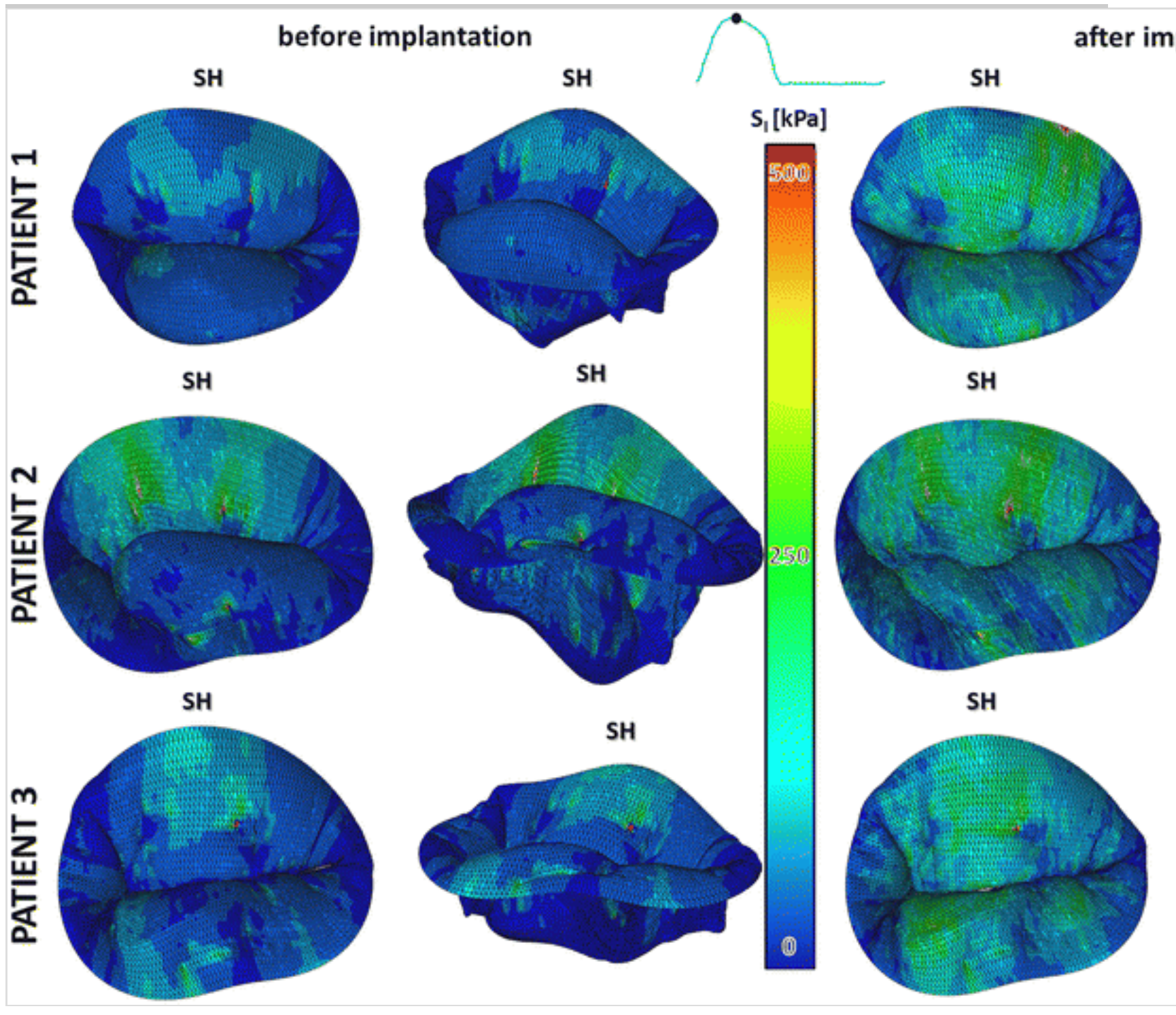

\section{Systolic Leaflet Stresses}

MV leaflets stress were analyzed at peak systole (Fig. 7): mechanical stresses increased on MV leaflets after MitraClip ${ }^{\circledR}$ procedure, although maintaining the same order of magnitude in a range between $100 \mathrm{kPa}$ and $500 \mathrm{kPa}$; moreover, mechanical stresses were increased on the A2-P2 of anterior and posterior mitral leaflets, i.e., where MItraClip ${ }^{\circledR}$ implantation was performed, as visible from the atrial view of each model.

\section{Figure 7}

Coaptation area (CoA) on the posterior leaflets on the three simulated MVs, in pathological conditions (left) and following Mitraclip ${ }^{\circledR}$ implantation (right). Green regions represent the $\mathrm{CoA}$ in pathological conditions, blue 
regions represent the extra extent recovered after Mitraclip ${ }^{\circledR}$ implantation

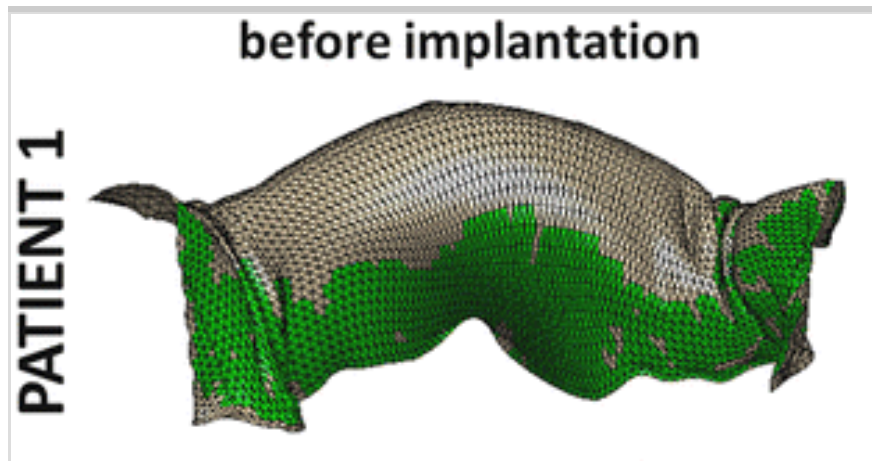

$$
\mathrm{CoA}=405.3 \mathrm{~mm}^{2}
$$
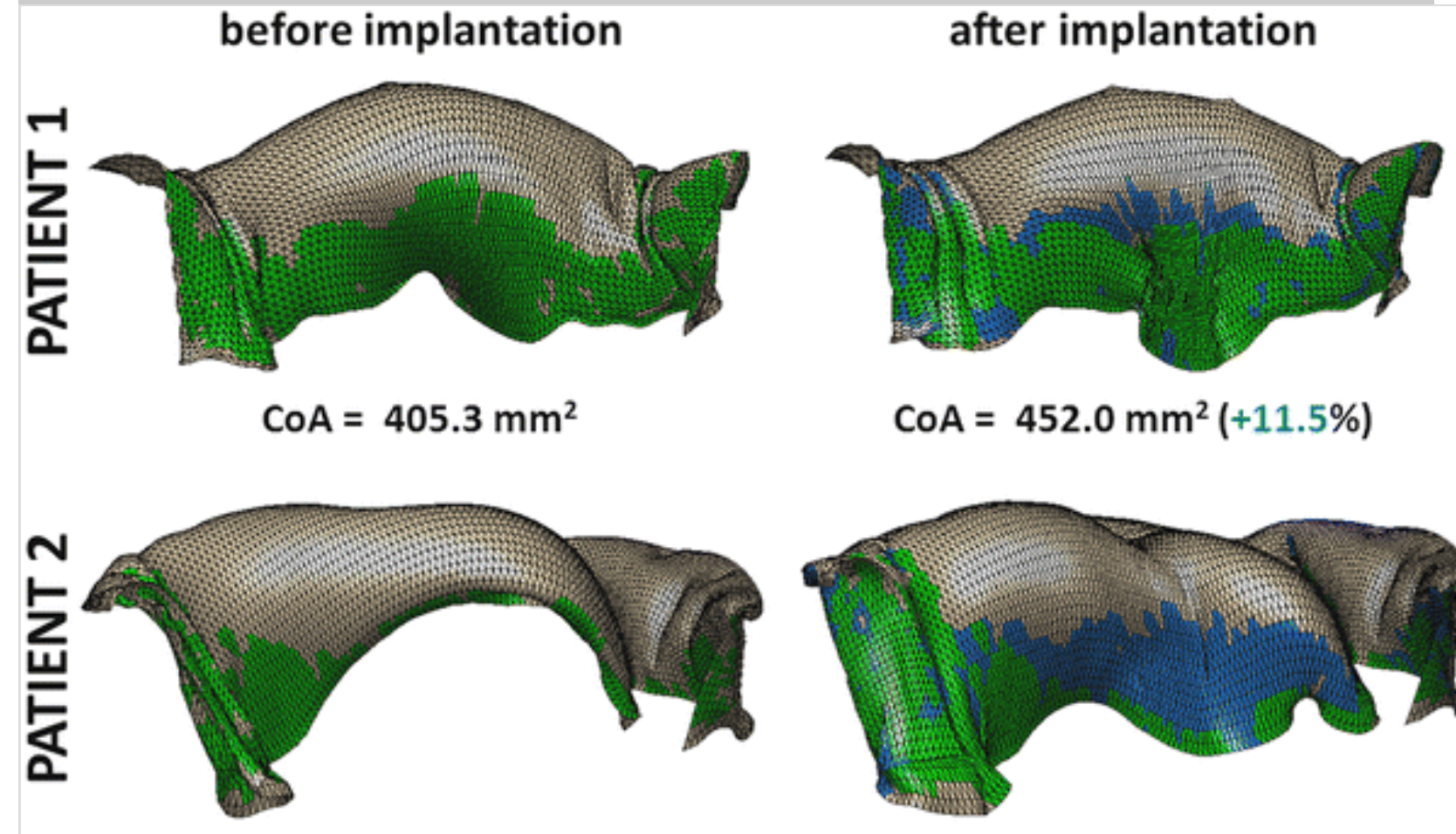

$$
\mathrm{COA}=555.1 \mathrm{~mm}^{2}
$$
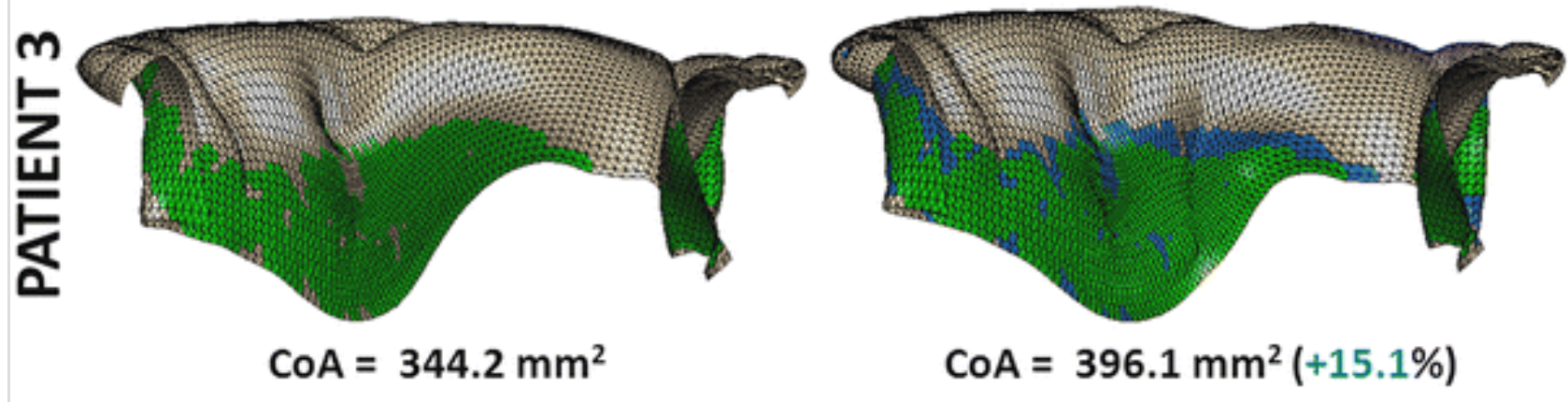

residual COA

recovered CoA

\section{Diastolic Leaflet Stresses}

Diastolic stresses were computed at maximal diastolic pressure (Fig. 8). In the pre-operative simulations, as expected, leaflets stresses were negligible. Simulated MitraClip ${ }^{\circledR}$ procedure induced a stress increase (in particular, e.g., up to $250 \mathrm{kPa}$ in patient 3) next to the region of insertion of the clip; these region of extra stresses extended from the free edge towards the annulus, however depending on the specific patient the effect in the annular region was different: negligible in patient 1 and patient 3 , and well evident in patient 2 , characterized by peak stresses higher than $300 \mathrm{kPa}$ next to the saddle horn.

\section{Figure 8}


Leaflets maximum principal stresses at early diastole in pathological conditions (left) and following Mitraclip ${ }^{\circledR}$ implantation (right), as seen from an atrial view

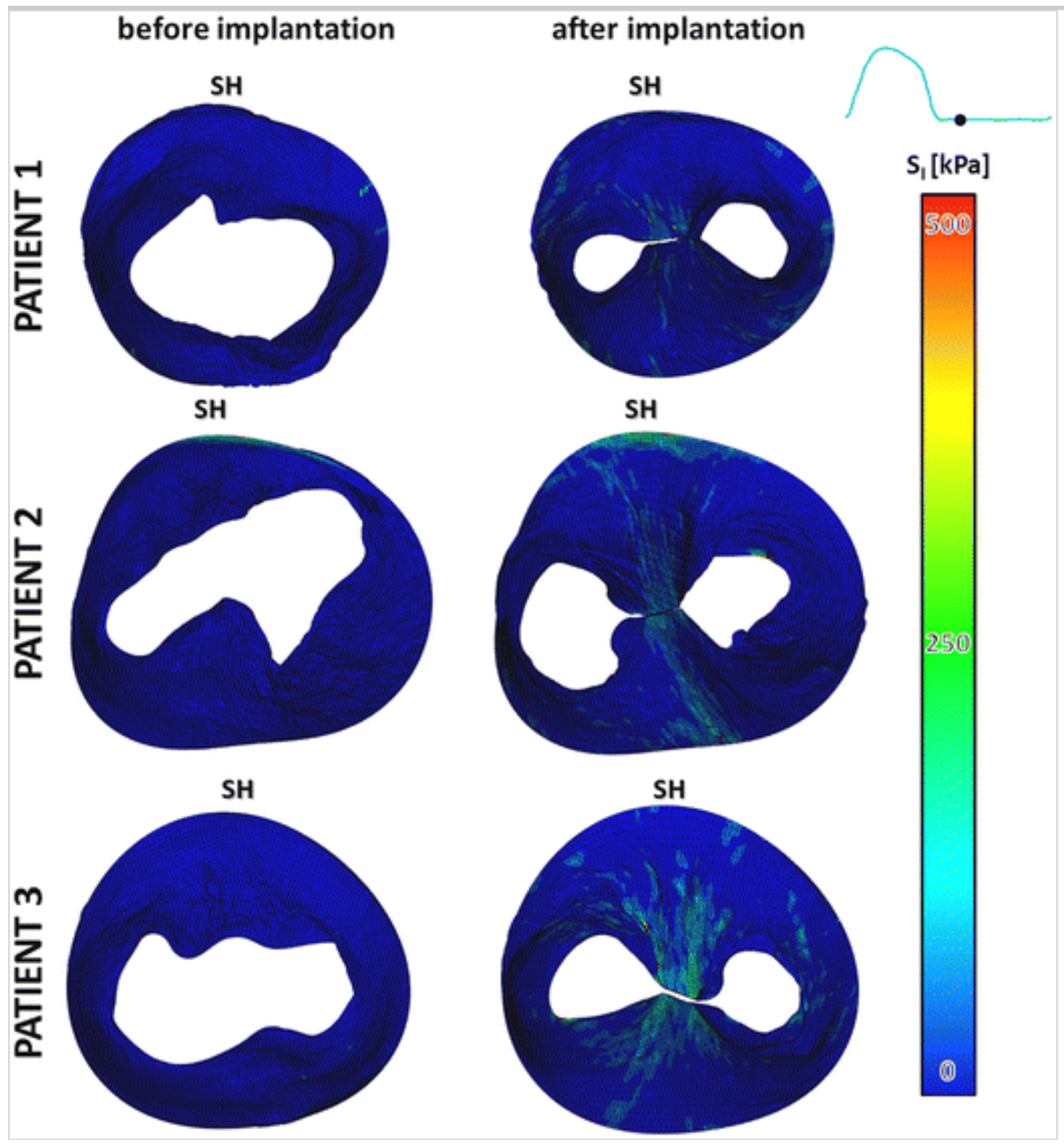

\section{Diastolic Orifice Area}

The orifice area computed in the different simulations are reported in Fig. 9. As reported by previous computational and experimental studies, the creation of a double-orifice induced a major reduction in effective orifice area, with respect the pre-operative configuration, which ranged from $32.8 \%$ (patient 2) to $62 \%$ (patient 1 ).

\section{Figure 9}

MV Orifice area (OA) available for blood flow in pathological conditions (black contour) and double orifice area (DOA) remaining after Mitraclip ${ }^{\circledR}$ 
implantation (red contour). Area are computed at early diastole and in 2D, i.e., projecting the leaflets free edge on the annulus least square plane

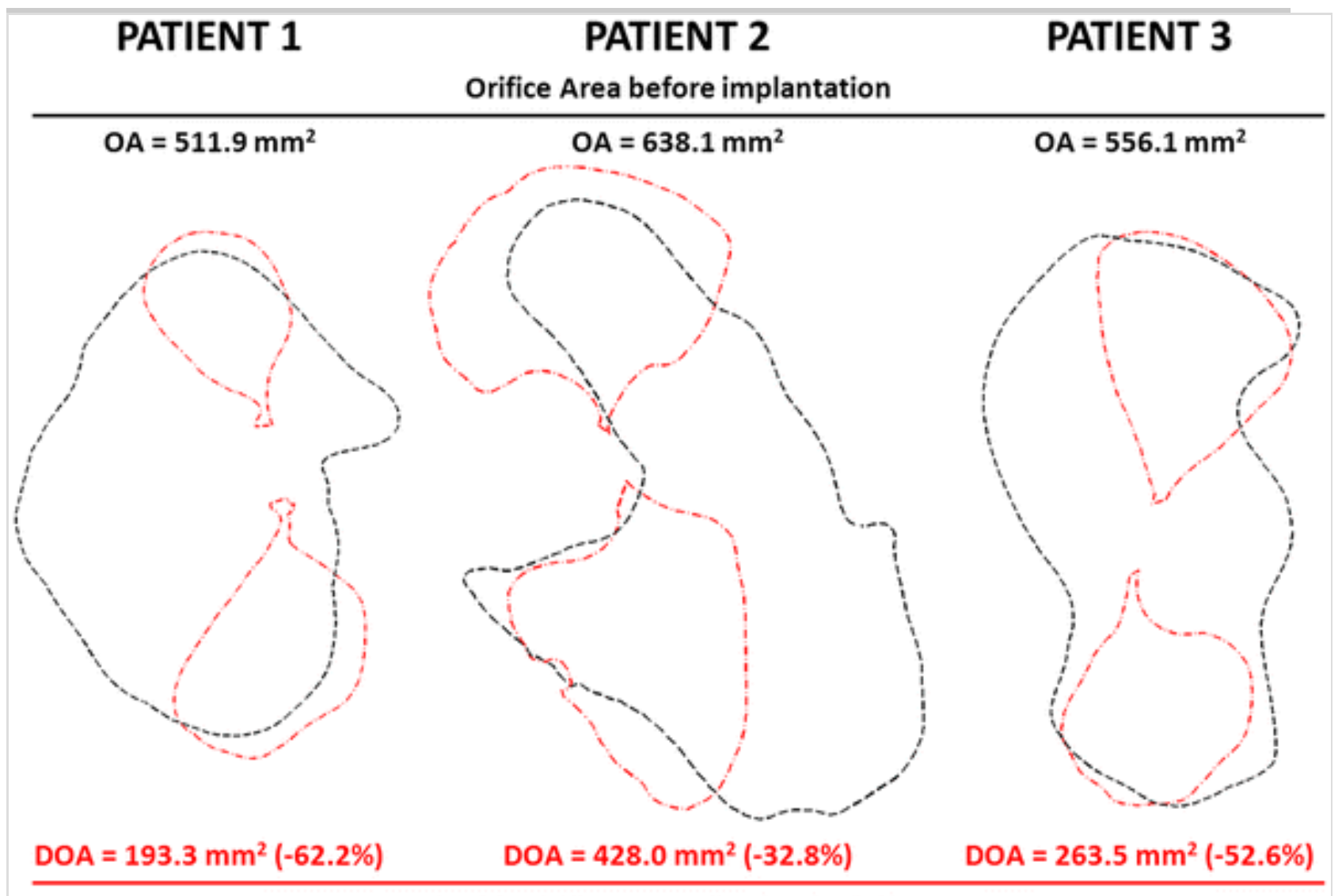

Double Orifice Area after implantation

\section{Discussion}

\section{Novelty of the Study}

Since the seminal study by Kunzeman et al., 38 finite element models have been increasingly adopted to better understand the effects of different mitral repair techniques, including annuloplasty, 12,39,68,80 chordal replacement, 62,63,70 Alfieri stitch, 14,79 and Mitraclip ${ }^{\circledR}$ implantation. 7,52

In this process, progressively more refined and realistic modeling approaches have been developed. Early studies used idealized models based on simplifying assumptions such as symmetrical MV geometry, static annulus and boundary conditions, and linear elastic tissues mechanical properties, e.g. Refs. 38, 79. Conversely, the most recent studies are characterized by realistic MV geometries based on clinical images, account for the dynamics of annulus and PMs, which can also be based on clinical images, and include the mathematical description of 
anisotropic and non-linear tissues mechanical properties. $63,68,70$

Yet, these advancements of the state of the art of MV computational modeling have not been exploited for the analysis of MV biomechanics following Mitraclip ${ }^{\circledR}$ implantation, despite the high clinical interest in this device. To the best of our knowledge, only two studies analyzed this specific clinical scenario by means of finite element modeling.

On the one hand, Avanzini and colleagues computed the distribution of leaflets stresses at peak systole and at end diastole following the implantation of the Mitraclip ${ }^{\circledR}$ device, through a finite element study aimed at providing indications of general validity. 7 However, despite the careful modeling of the device, their valve model represented an idealized and apparently physiological MV; this choice limited the possibility to realistically quantify the effects of the clip on MV continence, as well as on leaflets configuration and stresses. Also, their modeling approach suffered from most of the limitations characterizing the earliest studies in the field: (i) annular motion and PMs motion was neglected; (ii) leaflets tissue was assumed non-linear elastic but isotropic and with no difference between the two leaflets; (iii) chordae tendineae were not explicitly included into the model, but accounted for through a kinematic constraint applied to the leaflets free margin, implicitly assuming that the presence of the clip does not influence the position of leaflets free margin; (iv) both the anterior and posterior leaflet were modeled as symmetrical single cusps, with no continuity in the commissural region and with uniform thickness.

On the other hand, through a complementary approach, Mansi and colleagues developed a highly automated framework for the generation of MV 3D models from 3D ultrasound imaging, and for the subsequent patient-specific simulation of MV function in baseline conditions and following surgical repair. 63 The framework was aimed at aiding surgical planning, and its predictive potential was tested by simulating the implantation of a Mitraclip ${ }^{\circledR}$ device on a real patient and by comparing the computed outcome to the real one. This model was characterized by highly realistic geometry and kinematic boundary conditions, which were all derived directly from medical images, and by the detailed description of non-linear and anisotropic mechanical properties of MV leaflets, including the spatial variations in collagen fibers preferred direction within leaflets tissue. The only major simplification regarded the modeling of the clip, 
which was represented by a stiff spring that pulls together two nodes belonging to the free margin of the anterior and posterior leaflet, respectively; as a result, the effect of the clip is pointwise, rather than distributed on the non-negligible area grasped by the real device. Despite the level of sophistication of their modeling strategy, Mansi and coworkers focused their analysis on the computed post-intervention morphology of the valve, and on its consistency with the real one, and did not report any mechanical variable of interest.

Based on this state of the art, the present study is the first one that combines the morphological information provided by CMR with a reasonably detailed description of tissues mechanical properties and boundary kinematic constraints to provide a rather exhaustive analysis of the biomechanical effects of the Mitraclip ${ }^{\circledR}$ device, by means of geometrically realistic finite element models based on clinical imaging of pathological MVs that well represent the clinical indications for Mitraclip ${ }^{\circledR}$ implantation.

\section{On the Modeling Strategy}

Two aspects of the modeling approach herein presented represent a novelty with respect to previous studies of our research group, and may be worth being discussed in light of the current state of the art and of their impact on computed results.

The first aspect is the reconstruction of leaflets 3D geometry. As in Refs., 52,63,70 we developed a method to reconstruct the entire 3D geometry of MV leaflets from clinical imaging, whereas in the past we only accounted for few local measures of leaflets extent, and assumed their surface laying on a roughly conical surface. 68,70 Rim and colleagues reconstructed MV leaflets surface by means of non-uniform rational Bsplines that interpolate traced points in the 3D space. 63 That approach allows for capturing leaflets anatomy with great detail, but, if no smoothing is applied and no tolerance is accepted in the interpolation process, the quality of the resulting surface can be limited by the noise possibly affecting the interpolated raw data. Indeed, Rim and colleagues used input data from real time 3D trans-esophageal echocardiography, which allows for the clear identification of leaflets profile, thus reducing the noise affecting the corresponding traced profiles. As a result, the leaflets surface they reconstructed was characterized by detailed, local but 
smooth variations in curvature. On the other hand, for the sake of automation Mansi and co-workers used machine learning and statistical algorithms that yielded a very smooth leaflets surface, with less local geometrical details, 52 even though they also used 3D trans-esophageal echocardiography as a source of morphological data. In the present work, we developed a reconstruction method that differs from the two referred ones, and that provides leaflets surfaces with a level of detail that appears qualitatively closer to the one obtained in Ref. 63 rather than to the one observed in Ref. 67 Our method uses cubic splines to interpolate manually traced points on the CMR cut-planes, but these are subsequently sampled, and the obtained points are approximated via Fourier functions. This procedure implicitly filters noise affecting raw data; it was implemented because in CMR images leaflets are not visible as clearly as they are in transesophageal ultrasound images, and this uncertainty can introduce noise in the manually traced data. Yet this type of filtering allowed for capturing local changes in leaflet curvature and, in particular, the convexity towards the ventricle that characterizes the belly of the anterior leaflet when the valve is open; this feature is particularly relevant, given the impact of such convexity on the capability of the anterior leaflet to withstand systolic ventricular pressure without undergoing unrealistic bulging. 69 It might be worth noticing that due to the use of Fourier functions also to reconstruct the leaflet free margin profile, the indentations characterizing the commissures and paracommissures in real valves are less evident and characterized by a smoother shape in the reconstructed 3D model. This approximation is present generally in imagebased models, 52,63 owing to the need for raw data filtering, and is present even in models based on extremely well resolved images, such us micro computed tomography. 45 Nonetheless, we believe that this local geometrical approximation is not likely to affect the results of our computations, because of two reasons. First, commissural and paraccommisural regions are not affected by prolapse in our pathological models, nor are they involved by the clip simulation in the post-operative models. Second, despite the abovementioned approximation, the annulusto-free edge extent of the modeled leaflets in these regions was reasonably preserved as compared to the extent obtained from the raw data, the mismatch being always below $2 \mathrm{~mm}$, i.e., approximately 1.5 pixels. Of note, the preservation of the leaflet annulus-to-free edge extent allowed for the proper setting of the initial length of the modeled chordae tendineae inserted in these regions. This aspect is crucial, since a wrong setting of 
chordae initial length can heavily affect the computed closed configuration of the MV, as well explained in Ref. 52

The second aspect is the modelling the response of mitral leaflets, and of the anterior leaflet in particular. Although evidence from in vivo studies on ovine models shows that the anterior mitral leaflet is characterized by active contractile activity that makes its in vivo stiffness much greater than ex vivo stiffness, $35,36,71$ the vast majority of finite element models dealing with the simulation of MV in vivo behavior describe the response of the anterior leaflet as passive, based on stress-strain data from ex vivo tests. As a consequence, simulated MVs are often characterized by excessive bulging and prolapse even when they should mimic physiological conditions; these undesired features are partially avoided when active stiffening is accounted for by the adopted constitutive model. 65 In the present work, we adopted a passive, non-linear elastic and transversely isotropic model to describe both leaflets; however, we switched from the widely adopted constitutive model originally proposed by May-Newman 54 to the one recently adopted by Lee and colleagues. 45 Both models are based on the interpretation of leaflets microstructure as an elastic matrix reinforced by crimped preferentially oriented collagen fibers, are invariant-based, and use exponential functions of the 1st and 4th invariants of the right Cauchy-Green strain tensor. However, the model by May-Newman assumes that collagen fibers are perfectly aligned, whereas the model by Lee accounts for fiber dispersion through the parameter $\delta$. This difference reflects into a different behavior of the anterior leaflet when loaded by pressure, even when the constitutive parameters of the two models are identified by fitting of the same data: in our experience, when the model by May-Newman is adopted, the anterior leaflet is excessively extensible in the radial direction, i.e., perpendicularly to the fibers, undergoes unrealistic nominal radial strains well beyond 0.5 , and experiences unrealistic bulging. 68,81 Conversely, the use of the model by Lee reduced radial extensibility; this feature, together with the correct reconstruction of anterior leaflet diastolic geometric configuration, contributes to limiting the occurrence of undesired anterior leaflet bulging, confirming the impact of both initial geometry and mechanical properties on the computed systolic configuration reported by Stevanella et al. 69 Nonetheless, as described in the Limitations section, future efforts are mandatory to make the modeling of pathological human MV leaflets more realistic. 


\section{Clinical Implications of Biomechanical Analyses}

Performed simulations confirmed that, as for the surgical edge-to-edge repair, Mitraclip ${ }^{\circledR}$ implantation induces abnormal diastolic stresses, in particular next to the site of insertion. These are lower but comparable to leaflets stresses computed at peak systole, and are not characterized by dramatic local stress concentrations. This result appears consistent with evidence from histological analyses, 41,46 which reported that after in vivo the device is gradually encapsulated by connective tissue, which becomes progressively more organized in time. This process usually characterizes healing and foreign body response, and apparently is not related to stress-driven tissue remodeling.

Clinically relevant indications may be obtained by applying the modeling approach herein proposed to the analysis of suboptimal leaflets grasping and Mitraclip ${ }^{\circledR}$ positioning, as well as to gain insight into the clinical scenarios that are currently critical for this percutaneous procedure, such as functional MR and annular dilation. The latter may be of particular interest, since real systems for percutaneous annuloplasty of the posterior annulus are currently under evaluation, and the possibility to combine them with percutaneous edge-to-edge may become of clinical interest.

\section{Limitations of the Study}

Four main limitations of the present study should be stressed.

First, although based on realistic reconstruction of annulus and leaflets geometry, as well as of annulus and PMs motion, the 3D geometrical model of the MV here proposed has some limitations. The first one is the use of a paradigmatic space-dependent leaflet thickness, which was originally intended for healthy leaflets. 40 Also, chordae tendineae, which are not visible in CMR images, were modelled through a paradigmatic scheme, which was modified so to mimic the patient-specific chordal impairment and leaflet prolapse/flail. As recently shown, 64 the configuration of chordae tendineae has a rather relevant impact on the rest of the MV; hence, a finer patient-specific tuning of chordae tendineae may lead to better consistency with in vivo data.

Second, standard mechanical properties obtained from ex vivo testing of healthy porcine data were used. The properties of diseased human MV 
leaflets from old or relatively old patients, as the ones considered in the present study, are likely to be notably different from the one herein assumed. In particular, three aspects may cause such mismatch: interspecies differences, the effect of ageing, and the difference between healthy and diseased tissue. As regards the first two aspects, Pham and Sun tested the mechanical response of aged human mitral leaflets through biaxial testing, and showed that these were stiffer than porcine leaflets. They also pointed out that in individuals older than 65 the mechanical properties of mitral leaflets are not affected by age, but gradual stiffening of the leaflets may be observed when comparing leaflets from young adults to the ones of progressively elder people, given the decrease in glycosaminoglycan content and the progressive calcification associated to ageing. 59 As regards the third aspect, it has to be highlighted that degenerative disease alters the microstructure of MV leaflets and chordae tendineae, and hence the respective mechanical properties. As exhaustively detailed in the clinical literature of MV degenerative disease, 2 this pathological condition is characterized by a wide spectrum of scenarios, characterized by progressively more severe alterations of MV macroscopic morphology and tissue microstructure/mechanical properties. In the worst one, i.e., Barlow MV prolapse, the layered microstructure of mitral leaflet is completely altered: the spongiosa is abnormally thick and rich in glycosamminoglycans that infiltrate the fibrosa. At the same time, collagen bundles in the fibrosa would be disrupted. 53 These alterations would be paralleled by even more evident ones in the chordae tendineae. 26 However, we performed our simulations on MVs affected by FED, i.e., the simplest form of degenerative disease, usually associated with single chordal rupture and limited tissue degeneration. In FED-affected MVs the alterations of tissue microstructure are localilzed at few local points, usually at the insertion of ruptured/elongated native chordae. 53 In our pathological simulations, the diseased chordae were ruptured, and the corresponding site of insertion did not experience local load transfer from the chorda to the leaflet. In our repair simulations, the clip, placed at the site of valvular defect, likely distributed this load transfer. Hence, in this pilot study the computation of local strains/stresses may have been not dramatically affected by assuming healthy tissue mechanical properties. Based on these considerations, future work on the simulation of surgical repair of aged or diseased MVs will necessarily include the identification of more realistic constitutive parameters. 
Third, no thorough validation of our post-repair models could be performed, since the three patients selected for the study well represented a clinical scenario suitable for the adoption of the Mitraclip procedure, i.e., degenerative disease with no relevant annular dilation, but were treated with a different repair technique. Hence, no comparison between computed results and ground truth data was possible for the Mitraclip simulations. Instead, we checked for the consistency between the results of the simulations of MV pathological (i.e., pre-repair) closure and the corresponding pre-operative imaging data. Such consistency represents a necessary, but not sufficient, requirement to rely on the results of the repair simulations.

Fourth, the modeling approach herein presented is suitable to gain insight into the biomechanical effects of pathologies and repair techniques through models that include detailed geometrical information and kinematic boundary conditions. However, this approach could not be used as a tool to support surgical planning, owing to the excessive computational cost of our finite element simulations; the complete simulation of Mitraclip implantation and MV postoperative biomechanics throughout a complete cardiac cycle required about $6-8 \mathrm{~h}$, according to the elements number of each model, on an Intel Xeon $(2.93 \mathrm{GHz})$ workstation with 8 CPUs.

\section{Conflict of interest}

All of the authors declare that they do not have any conflict of interest and financial and personal relationships with other people or organizations that could inappropriately influence their work.

\section{Statement of Animal Studies}

No animal studies were carried out by the authors for this article.

\section{Statement of Human Studies}

All procedures followed were in accordance with the ethical standards of the responsible committee on human experimentation (institutional and national) and with the Helsinki Declaration of 1975, as revised in 2000. Informed consent was obtained from all patients for being included in the study. 


\section{References}

1. Abbott Laboratories. Electronic instructions for use for the MitraClip(R) clip delivery system.

http://www.abbottvascular.com/static/cms_workspace/pdf/ifu/structural_hear 2014. Accessed 12 June 2014.

2. Adams, D. H., A. C. Anyanwu, P. B. Rahmanian, and F. Filsoufi. Current concepts in mitral valve repair for degenerative disease. Heart Fail. Rev. 11(3):241-257, 2006. doi:10.1007/s10741-006-0103-7.

3. Al-Atassi, T., T. Malas, T. Mesana, and V. Chan. Mitral valve interventions in heart failure. Curr. Opin. Cardiol. 29(2):192-197, 2014.

4. Alfieri, O., and M. De Bonis. The role of the edge-to-edge repair in the surgical treatment of mitral regurgitation. J. Card. Surg. 25(5):536$541,2010$.

5. Alfieri, O., F. Maisano, M. De Bonis, P. L. Stefano, L. Torracca, M. Oppizzi, et al. The double-orifice technique in mitral valve repair: a simple solution for complex problems. J. Thorac. Cardiovasc. Surg. 122(4):674-681, 2001.

6. Alfieri, O., J. A. Elefteriades, R. J. Chapolini, R. Steckel, W. J. Allen, S. W. Reed, et al. Novel suture device for beating-heart mitral leaflet approximation. Ann. Thorac. Surg. 74(5):1488-1493, 2002.

7. Avanzini, A., G. Donzella, and L. Libretti. Functional and structural effects of percutaneous edge-to-edge double-orifice repair under cardiac cycle in comparison with suture repair. Proc. Inst. Mech. Eng. H. 225(10):959-971, 2011.

8. Bhudia, S. K., P. M. McCarthy, N. G. Smedira, B. K. Lam, J. Rajeswaran, and E. H. Blackstone. Edge-to-edge (Alfieri) mitral repair: results in diverse clinical settings. Ann. Thorac. Surg. 77(5):1598-1606, 2004.

9. Bonow, R. O., B. A. Carabello, K. Chatterjee, A. C. De Leon, Jr., D. 
P. Faxon, M. D. Freed, et al. Focused update incorporated into the ACC/AHA 2006 guidelines for the management of patients with valvular heart disease: a report of the American College of Cardiology/American Heart Association Task Force on Practice Guidelines (Writing Committee to Revise the 1998 Guidelines for the Management of Patients With Valvular Heart Disease): endorsed by the Society of Cardiovascular Anesthesiologists, Society for Cardiovascular Angiography and Interventions, and Society of Thoracic Surgeons. Circulation. 118(15):26, 2008.

10. Braun, D., H. Lesevic, M. Orban, F. Michalk, P. Barthel, K. Hoppe, et al. Percutaneous edge-to-edge repair of the mitral valve in patients with degenerative versus functional mitral regurgitation. Catheter. Cardiovasc. Interv. 84(1):137-146, 2014. doi:10.1002/ccd.25331.

11. Braunberger, E., A. Deloche, A. Berrebi, F. Abdallah, J. A. Celestin, P. Meimoun, et al. Very long-term results (more than 20 years) of valve repair with carpenter's techniques in nonrheumatic mitral valve insufficiency. Circulation. 104(12 Suppl 1):I8-I11, 2001.

12. Choi, A., Y. Rim, J. S. Mun, and H. Kim. A novel finite elementbased patient-specific mitral valve repair: virtual ring annuloplasty. Biomed. Mater. Eng. 24(1):341-347, 2014. doi:10.3233/bme-130816.

13. Croft, L. R., J. H. Jimenez, R. C. Gorman, J. H. Gorman, 3rd, and A. P. Yoganathan. Efficacy of the edge-to-edge repair in the setting of a dilated ventricle: an in vitro study. Ann. Thorac. Surg. 84(5):15781584, 2007. doi:10.1016/j.athoracsur.2007.05.086.

14. Dal Pan, F., G. Donzella, C. Fucci, and M. Schreiber. Structural effects of an innovative surgical technique to repair heart valve defects. J. Biomech. 38(12):2460-2471, 2005. doi:10.1016/j.jbiomech.2004.10.005.

15. Denti, P., F. Maisano, and O. Alfieri. Devices for mitral valve repair. J. Cardiovasc. Transl. Res. 7(3):266-281, 2014.

16. Dimasi, A., E. Cattarinuzzi, M. Stevanella, C. Conti, E. Votta, F. Maffessanti, et al. Influence of mitral valve anterior leaflet in vivo 
shape on left ventricular ejection. Cardiovasc. Eng. Technol. 3(4):388401, 2012. doi:10.1007/s13239-012-0105-7.

17. Du, D., S. Jiang, Z. Wang, Y. Hu, and Z. He. Effects of suture position on left ventricular fluid mechanics under mitral valve edge-toedge repair. Biomed. Mater. Eng. 24(1):155-161, 2014. doi:10.3233/bme-130795.

18. Ducas, R. A., C. W. White, A. W. Wassef, A. Farag, K. M. Bhagirath, D. H. Freed, et al. Functional mitral regurgitation: current understanding and approach to management. Can. J. Cardiol. 30(2):173-180, 2014.

19. Falk, V., J. Seeburger, M. Czesla, M. A. Borger, J. Willige, T. Kuntze, et al. How does the use of polytetrafluoroethylene neochordae for posterior mitral valve prolapse (loop technique) compare with leaflet resection? A prospective randomized trial. J. Thorac. Cardiovasc. Surg. 136(5):1205, 2008. doi:10.1016/j.jtcvs.2008.07.028; discussion-6.

20. Feldman, T., and A. Young. Percutaneous approaches to valve repair for mitral regurgitation. J. Am. Coll. Cardiol. 63(20):2057-2068, 2014. doi:10.1016/j.jacc.2014.01.039.

21. Feldman, T., S. Kar, M. Rinaldi, P. Fail, J. Hermiller, R. Smalling, et al. Percutaneous mitral repair with the MitraClip system: safety and midterm durability in the initial EVEREST (Endovascular Valve Edgeto-Edge REpair Study) cohort. J. Am. Coll. Cardiol. 54(8):686-694, 2009. doi:10.1016/j.jacc.2009.03.077.

22. Feldman, T., E. Foster, D. D. Glower, S. Kar, M. J. Rinaldi, P. S. Fail, et al. Percutaneous repair or surgery for mitral regurgitation. $N$. Engl. J. Med. 364(15):1395-1406, 2011. doi:10.1056/NEJMoa1009355.

23. Filsoufi, F., and A. Carpentier. Principles of reconstructive surgery in degenerative mitral valve disease. Semin. Thorac. Cardiovasc. Surg. 19(2):103-110, 2007.

24. Fucci, C., L. Sandrelli, A. Pardini, L. Torracca, M. Ferrari, and O. 
Alfieri. Improved results with mitral valve repair using new surgical techniques. Eur. J. Cardiothorac. Surg. 9(11):621-626, 1995.

25. Gould, S. T., S. Srigunapalan, C. A. Simmons, and K. S. Anseth. Hemodynamic and cellular response feedback in calcific aortic valve disease. Circ. Res. 113(2):186-197, 2013.

doi:10.1161/circresaha.112.300154.

26. Grande-Allen, K. J., B. P. Griffin, N. B. Ratliff, D. M. Cosgrove, and I. Vesely. Glycosaminoglycan profiles of myxomatous mitral leaflets and chordae parallel the severity of mechanical alterations. $J$. Am. Coll. Cardiol. 42(2):271-277, 2003.

27. Hasegawa, H., Y. Araki, A. Usui, J. Yokote, S. Saito, H. Oshima, et al. Mitral valve motion after performing an edge-to-edge repair in an isolated swine heart. J. Thorac. Cardiovasc. Surg. 136(3):590-596, 2008. doi:10.1016/j.jtcvs.2008.03.050.

28. He, Z., B. Gao, S. Bhattacharya, T. Harrist, S. Mathew, and W. Sun. In vitro stretches of the mitral valve anterior leaflet under edge-toedge repair condition. J. Biomech. Eng. 131(11):111012, 2009. doi:10.1115/1.4000111.

29. Hilberath, J. N., H. K. Eltzschig, S. K. Shernan, A. H. Worthington, S. F. Aranki, and M. Nowak-Machen. Intraoperative evaluation of transmitral pressure gradients after edge-to-edge mitral valve repair. Plos One. 8(9):e73617, 2013. doi:10.1371/journal.pone.0073617.

30. Hu, Y., L. Shi, S. Parameswaran, S. Smirnov, and Z. He. Left ventricular vortex under mitral valve edge-to-edge repair. Cardiovasc. Eng. Technol. 1(4):235-243, 2010. doi:10.1007/s13239-010-0022-6.

31. Huang, H. Y., J. Liao, and M. S. Sacks. In-situ deformation of the aortic valve interstitial cell nucleus under diastolic loading. J. Biomech. Eng. 129(6):880-889, 2007.

32. Jimenez, J. H., J. Forbess, L. R. Croft, L. Small, Z. He, and A. P. Yoganathan. Effects of annular size, transmitral pressure, and mitral flow rate on the edge-to-edge repair: an in vitro study. Ann. Thorac. 
Surg. 82(4):1362-1368, 2006. doi:10.1016/j.athoracsur.2006.05.008.

33. Kim, W. Y., P. G. Walker, E. M. Pedersen, J. K. Poulsen, S. Oyre, $\mathrm{K}$. Houlind, et al. Left ventricular blood flow patterns in normal subjects: a quantitative analysis by three-dimensional magnetic resonance velocity mapping. J. Am. Coll. Cardiol. 26(1):224-238, 1995.

34. Krishnamurthy, G., D. B. Ennis, A. Itoh, W. Bothe, J. C. Swanson, M. Karlsson, et al. Material properties of the ovine mitral valve anterior leaflet in vivo from inverse finite element analysis. Am. J. Physiol. Heart Circ. Physiol. 295(3):H1141-H1149, 2008.

35. Krishnamurthy, G., A. Itoh, J. C. Swanson, W. Bothe, M. Karlsson, E. Kuhl, et al. Regional stiffening of the mitral valve anterior leaflet in the beating ovine heart. J. Biomech. 42(16):2697-2701, 2009. doi:10.1016/j.jbiomech.2009.08.028.

36. Krishnamurthy, G., A. Itoh, J. C. Swanson, D. C. Miller, and N. B. Ingels, Jr. Transient stiffening of mitral valve leaflets in the beating heart. Am. J. Physiol. Heart Circ. Physiol. 298(6):H2221-H2225, 2010. doi:10.1152/ajpheart.00215.2010.

37. Kunzelman, K. S., and R. P. Cochran. Mechanical properties of basal and marginal mitral valve chordae tendineae. ASAIO Trans. 36(3):M405-M408, 1990.

38. Kunzelman, K. S., R. P. Cochran, C. Chuong, W. S. Ring, E. D. Verrier, and R. D. Eberhart. Finite element analysis of the mitral valve. J. Heart Valve Dis. 2(3):326-340, 1993.

39. Kunzelman, K. S., M. S. Reimink, and R. P. Cochran. Flexible versus rigid ring annuloplasty for mitral valve annular dilatation: a finite element model. J. Heart Valve Dis. 7(1):108-116, 1998.

40. Kunzelman, K. S., D. R. Einstein, and R. P. Cochran. Fluidstructure interaction models of the mitral valve: function in normal and pathological states. Philos. Trans. R. Soc. Lond. B Biol. Sci. 362(1484):1393-1406, 2007. doi:10.1098/rstb.2007.2123. 
41. Ladich, E., M. B. Michaels, R. M. Jones, E. McDermott, L. Coleman, J. Komtebedde, et al. Pathological healing response of explanted MitraClip devices. Circulation. 123(13):1418-1427, 2011. doi:10.1161/circulationaha.110.978130.

42. Lam, J. H., N. Ranganathan, E. D. Wigle, and M. D. Silver. Morphology of the human mitral valve. I. Chordae tendineae: a new classification. Circulation. 41(3):449-458, 1970.

43. Latif, N., P. Sarathchandra, P. M. Taylor, J. Antoniw, and M. H. Yacoub. Molecules mediating cell-ECM and cell-cell communication in human heart valves. Cell Biochem. Biophys. 43(2):275-287, 2005. doi:10.1385/cbb:43:2:275.

44. Lau, K. D., V. Diaz-Zuccarini, P. Scambler, and G. Burriesci. Fluid-structure interaction study of the edge-to-edge repair technique on the mitral valve. J. Biomech. 44(13):2409-2417, 2011. doi:10.1016/j.jbiomech.2011.06.030.

45. Lee, C. H., R. Amini, R. C. Gorman, J. H. Gorman, 3rd, and M. S. Sacks. An inverse modeling approach for stress estimation in mitral valve anterior leaflet valvuloplasty for in vivo valvular biomaterial assessment. J. Biomech. 47(9):2055-2063, 2014.

doi:10.1016/j.jbiomech.2013.10.058.

46. Luk, A., J. Butany, E. Ahn, J. I. Fann, F. St Goar, T. Thornton, et al. Mitral repair with the Evalve MitraClip device: histopathologic findings in the porcine model. Cardiovasc. Pathol. 18(5):279-285, 2009. doi:10.1016/j.carpath.2008.07.001.

47. Maisano, F., L. Torracca, M. Oppizzi, P. L. Stefano, G. D’Addario, G. La Canna, et al. The edge-to-edge technique: a simplified method to correct mitral insufficiency. Eur. J. Cardiothorac. Surg. 13(3):240-245, 1998.

48. Maisano, F., A. Redaelli, G. Pennati, R. Fumero, L. Torracca, and O. Alfieri. The hemodynamic effects of double-orifice valve repair for mitral regurgitation: a 3D computational model. Eur. J. Cardiothorac. Surg. 15(4):419-425, 1999. 
49. Maisano, F., J. J. Schreuder, M. Oppizzi, B. Fiorani, C. Fino, and O. Alfieri. The double-orifice technique as a standardized approach to treat mitral regurgitation due to severe myxomatous disease: surgical technique. Eur. J. Cardiothorac. Surg. 17(3):201-205, 2000.

50. Maisano, F., A. Caldarola, A. Blasio, M. De Bonis, G. La Canna, and O. Alfieri. Midterm results of edge-to-edge mitral valve repair without annuloplasty. J. Thorac. Cardiovasc. Surg. 126(6):1987-1997, 2003.

51. Maisano, F., G. La Canna, A. Colombo, and O. Alfieri. The evolution from surgery to percutaneous mitral valve interventions: the role of the edge-to-edge technique. J. Am. Coll. Cardiol. 58(21):2174$2182,2011$.

52. Mansi, T., I. Voigt, B. Georgescu, X. Zheng, E. A. Mengue, M. Hackl, et al. An integrated framework for finite-element modeling of mitral valve biomechanics from medical images: application to MitralClip intervention planning. Med. Image Anal. 16(7):1330-1346, 2012. doi:10.1016/j.media.2012.05.009.

53. Matsumaru, I., K. Eishi, K. Hashizume, H. Kawano, A. Tsuneto, and T. Hayashi. Clinical and pathological features of degenerative mitral valve disease: billowing mitral leaflet versus fibroelastic deficiency. Ann. Thorac. Cardiovasc. Surg. 27:27, 2013.

54. May-Newman, K., and F. C. Yin. A constitutive law for mitral valve tissue. J. Biomech. Eng. 120(1):38-47, 1998.

55. Morales, D. L., J. D. Madigan, A. F. Choudhri, M. R. Williams, D. N. Helman, J. B. Elder, et al. Development of an off bypass mitral valve repair. Heart Surg. Forum. 2(2):115-120, 1999.

56. Naqvi, T. Z., M. Buchbinder, D. Zarbatany, J. Logan, M. Molloy, G. Balke, et al. Beating-heart percutaneous mitral valve repair using a transcatheter endovascular suturing device in an animal model.

Catheter. Cardiovasc. Interv. 69(4):525-531, 2007.

doi:10.1002/ccd.21029. 
57. Nielsen, S. L., T. A. Timek, D. T. Lai, G. T. Daughters, D. Liang, J. M. Hasenkam, et al. Edge-to-edge mitral repair: tension on the approximating suture and leaflet deformation during acute ischemic mitral regurgitation in the ovine heart. Circulation. 104(12 Suppl 1):I29-I35, 2001.

58. Nkomo, V. T., J. M. Gardin, T. N. Skelton, J. S. Gottdiener, C. G. Scott, and M. Enriquez-Sarano. Burden of valvular heart diseases: a population-based study. Lancet 368(9540):1005-1011, 2006.

59. Pham, T., and W. Sun. Material properties of aged human mitral valve leaflets. J. Biomed. Mater. Res. A. 102(8):2692-2703, 2014. doi:10.1002/jbm.a.34939.

60. Rabbah, J. P., A. W. Siefert, E. M. Spinner, N. Saikrishnan, and A. P. Yoganathan. Peak mechanical loads induced in the in vitro edge-toedge repair of posterior leaflet flail. Ann. Thorac. Surg. 94(5):14461453, 2012. doi:10.1016/j.athoracsur.2012.05.024.

61. Reichenspurner, H., W. Schillinger, S. Baldus, J. Hausleiter, C. Butter, U. Schaefer, et al. Clinical outcomes through 12 months in patients with degenerative mitral regurgitation treated with the MitraClip(R) device in the ACCESS-EUrope Phase I trial. Eur. J. Cardiothorac. Surg. 44(4):e280-e288, 2013. doi:10.1093/ejcts/ezt321.

62. Reimink, M. S., K. S. Kunzelman, and R. P. Cochran. The effect of chordal replacement suture length on function and stresses in repaired mitral valves: a finite element study. J. Heart Valve Dis. 5(4):365-375, 1996.

63. Rim, Y., S. T. Laing, D. D. McPherson, and H. Kim. Mitral valve repair using ePTFE sutures for ruptured mitral chordae tendineae: a computational simulation study. Ann. Biomed. Eng. 42(1):139-148, 2014.

64. Siefert, A. W., J. P. Rabbah, E. L. Pierce, K. S. Kunzelman, and A. P. Yoganathan. Quantitative evaluation of annuloplasty on mitral valve chordae tendineae forces to supplement surgical planning model development. Cardiovasc. Eng. Technol. 5(1):35-43, 2014. 
doi:10.1007/s13239-014-0175-9.

65. Skallerud, B., V. Prot, and I. S. Nordrum. Modeling active muscle contraction in mitral valve leaflets during systole: a first approach. Biomech. Model. Mechanobiol. 10(1):11-26, 2011. doi:10.1007/s10237-010-0215-9.

66. Solomon, N. A., S. K. Pranav, D. Naik, and S. Sukumaran. Importance of preservation of chordal apparatus in mitral valve replacement. Expert. Rev. Cardiovasc. Ther. 4(2):253-261, 2006. doi:10.1586/14779072.4.2.253.

67. Stevanella, M., E. Votta, and A. Redaelli. Mitral valve finite element modeling: implications of tissues' nonlinear response and annular motion. J. Biomech. Eng. 131(12):4000107, 2009.

68. Stevanella, M., F. Maffessanti, C. Conti, E. Votta, A. Arnoldi, M. Lombardi, et al. Mitral valve patient-specific finite element modeling from cardiac MRI: application to an annuloplasty procedure. Cardiovasc. Eng. Technol. 2(2):66-76, 2011. doi:10.1007/s13239-0100032-4.

69. Stevanella, M., G. Krishnamurthy, E. Votta, J. C. Swanson, A. Redaelli, and N. B. Ingels, Jr. Mitral leaflet modeling: importance of in vivo shape and material properties. J. Biomech. 44(12):2229-2235, 2011.

70. Sturla, F., F. Onorati, E. Votta, K. Pechlivanidis, M. Stevanella, A. D. Milano, et al. Is it possible to assess the best mitral valve repair in the individual patient? Preliminary results of a finite element study from magnetic resonance imaging data. J. Thorac. Cardiovasc. Surg. 148(3):1025-1034, 2014. doi:10.1016/j.jtcvs.2014.05.071; discussion 34.

71. Swanson, J. C., G. Krishnamurthy, A. Itoh, J. P. Kvitting, W. Bothe, D. Craig Miller, et al. Multiple mitral leaflet contractile systems in the beating heart. J. Biomech. 44(7):1328-1333, 2011.

doi:10.1016/j.jbiomech.2011.01.006. 
72. Tan, H., S. Biechler, L. Junor, M. J. Yost, D. Dean, J. Li, et al. Fluid flow forces and rhoA regulate fibrous development of the atrioventricular valves. Dev. Biol. 374(2):345-356, 2013. doi:10.1016/j.ydbio.2012.11.023.

73. Taramasso, M., P. Denti, N. Buzzatti, M. De Bonis, G. La Canna, A. Colombo, et al. Mitraclip therapy and surgical mitral repair in patients with moderate to severe left ventricular failure causing functional mitral regurgitation: a single-centre experience. Eur. J. Cardiothorac. Surg. 42(6):920-926, 2012. doi:10.1093/ejcts/ezs294.

74. Thayer, P., K. Balachandran, S. Rathan, C. H. Yap, S. Arjunon, H. Jo, et al. The effects of combined cyclic stretch and pressure on the aortic valve interstitial cell phenotype. Ann. Biomed. Eng. 39(6):16541667, 2011.

75. Timek, T. A., S. L. Nielsen, D. Liang, D. T. Lai, P. Dagum, G. T. Daughters, et al. Edge-to-edge mitral repair: gradients and threedimensional annular dynamics in vivo during inotropic stimulation. Eur. J. Cardiothorac. Surg. 19(4):431-437, 2001.

76. Timek, T. A., S. L. Nielsen, D. T. Lai, F. A. Tibayan, D. Liang, F. Rodriguez, et al. Edge-to-edge mitral valve repair without ring annuloplasty for acute ischemic mitral regurgitation. Circulation. 108(Suppl 1):II122-II127, 2003. doi:10.1161/01.cir.0000087943.76135.fd.

77. Timek, T. A., S. L. Nielsen, D. T. Lai, F. Tibayan, D. Liang, G. T. Daughters, et al. Mitral annular size predicts Alfieri stitch tension in mitral edge-to-edge repair. J. Heart Valve Dis. 13(2):165-173, 2004.

78. Vahanian, A., O. Alfieri, F. Andreotti, M. J. Antunes, G. BaronEsquivias, H. Baumgartner, et al. Guidelines on the management of valvular heart disease (version 2012). The Joint Task Force on the Management of Valvular Heart Disease of the European Society of Cardiology (ESC) and the European Association for Cardio-Thoracic Surgery (EACTS). G. Ital. Cardiol. 14(3):167-214, 2013.

79. Votta, E., F. Maisano, M. Soncini, A. Redaelli, F. M. Montevecchi, 
and O. Alfieri. 3-D computational analysis of the stress distribution on the leaflets after edge-to-edge repair of mitral regurgitation. J. Heart Valve Dis. 11(6):810-822, 2002.

80. Votta, E., F. Maisano, S. F. Bolling, O. Alfieri, F. M. Montevecchi, and A. Redaelli. The Geoform disease-specific annuloplasty system: a finite element study. Ann. Thorac. Surg. 84(1):92-101, 2007. doi:10.1016/j.athoracsur.2007.03.040.

81. Votta, E., T. B. Le, M. Stevanella, L. Fusini, E. G. Caiani, A. Redaelli, et al. Toward patient-specific simulations of cardiac valves: state-of-the-art and future directions. J. Biomech. 46(2):217-228, 2013.

82. Webb, J. G., F. Maisano, A. Vahanian, B. Munt, T. Z. Naqvi, R. Bonan, et al. Percutaneous suture edge-to-edge repair of the mitral valve. EuroIntervention 5(1):86-89, 2009.

83. Whitlow, P. L., T. Feldman, W. R. Pedersen, D. S. Lim, R. Kipperman, R. Smalling, et al. Acute and 12-month results with catheter-based mitral valve leaflet repair: the EVEREST II (Endovascular Valve Edge-to-Edge Repair) High Risk Study. J. Am. Coll. Cardiol. 59(2):130-139, 2012. doi:10.1016/j.jacc.2011.08.067. 